$\begin{array}{ll}\text { Research Square } & \begin{array}{l}\text { Preprints are preliminary reports that have not undergone peer review. } \\ \text { They should not be considered conclusive, used to inform clinical practice, } \\ \text { or referenced by the media as validated information. }\end{array}\end{array}$

\title{
The Retrospective Study of Prevalence and Associated Risk Factors of Diseases and Others Clinical Conditions Diagnosed in Dogs and Cats in Douala City, Cameroon
}

Justin Kouamo

School of Veterinary Medecine and Sciences

Alain Gildas Dongmo Kana ( $\sim$ kanadongmo1@gmail.com )

Faculty of Agronomy and Agricultural Sciences, University of Dschang https://orcid.org/0000-0002-9396-7370

Carène Chéryle Zebaze Dongmo

School of Veterinary Medicine and Sciences, University of Ngaoundere

Research article

Keywords: Prevalence, epidemiology, disease, clinical condition, dog and cat, Cameroon

Posted Date: July 17th, 2020

DOI: https://doi.org/10.21203/rs.3.rs-43889/v1

License: (c) (i) This work is licensed under a Creative Commons Attribution 4.0 International License. Read Full License

Version of Record: A version of this preprint was published at Revue Vétérinaire Clinique on May 1st, 2021. See the published version at https://doi.org/10.1016/j.anicom.2021.01.001. 


\section{Abstract}

Background: A good knowledge of the epidemiology of diseases conditions of pet dogs and cats is essential for their prevention and control. Over a period of 5 years (2014 to 2019), a retrospective study was undertaken to establish the prevalence of clinical diseases and others clinical disorders in dogs and cats in Douala city, Cameroon, based on sex, breeds and age. Data were collected from clinical case records of dogs and cats admitted in six private veterinary clinics and analyzed using Chi-Square test statistics.

Results: A total of 2397 clinical conditions were recorded, among them dogs and cats were 2222 (92.7\%) and 175 (7.3\%), respectively. According to system affected, digestive disorders were found to account significantly $(P<0.0001)$ for $58.3 \%$ of the diagnosed cases, followed by skin disorders (13.5\%) and musculoskeletal disorders (13.1\%). Fourty-six (46) types of clinical diseases were recorded involving nonspecific diseases (dogs $25.1 \%$ and cats 19.4\%), viral diseases (dogs 16.8\%), parasitic diseases (dogs $12.7 \%$ and cats $9.7 \%$ ), protozoan diseases (dogs $2.7 \%$ ) and bacterial/fungal diseases (dogs 1.4\%). For dogs, the most occurring clinical diseases comprised parvovirosis (16\%), helminthoses (8.2\%), gastroenteritis (7.8\%) and poisoning (7.8\%), whereas pet cats showed highest prevalence of helminthoses (9.7\%), poisoning (5.1\%) and dermatitis (2.9\%). The sex-wise highest prevalence was observed in male (dogs $67 \%$ and cats $59.4 \%$ ) than female (dogs $33 \%$ and cats $40.6 \%)(p=0.009)$. Of the dog breeds, Cross breeds ( $31.7 \%$ ), local breeds (24.02\%) and German shepherd (13.1\%) were mostly affected, while highest clinical diseases were found 96 (54.9\%) in the local cat breed counterpart. Incidence of diseases were more in puppies (68.7\%) and in young cats (75.5\%) compare with other age groups.

Conclusions: Most of the clinical diseases/conditions recorded from this study were avoidable cases and they have greater public health significance, demanding veterinary support in order to prevent the risk of zoonosis. This study provides useful information about the major health problems of dogs and cats in Douala City. These results might help to instruct pet owners on preventive measures and to develop supplementary effective disease management and control strategies against pet animal diseases.

\section{Background}

The keeping and rearing of pet animals are common throughout the world, with dogs and cats being the most widespread species [1]. Companion animals, particularly dogs and cats offer individuals and the community significant benefits such as physiological support and companionship. In addition, they can play with children, guard the house and alerts the owner from any adverse situation, providing a psychological benefits and good health support [2, 3]. Sometimes pets are used as gift to someone and as economic purpose. People living with a pet animal have been reported to have fewer doctors' consultations and longer endurance following heart illness compared to non-pet owners [4]. Today, there is much tendency of using pet animals in therapeutic settings. Indeed, companion animals have an ability to stimulate non-threatening and playful contact, thus their faculty to act as social catalysts [1]. Close human interactions with companion animals provides emotional support and influence wellbeing significantly. They may reduce perception of stressful related illness, protect against anxiety, and may improve recovery from severe disorders like stroke and myocardial infarction [5]. Pets have also proven to play different roles such as guide for handicapped individual, offer assistance to the disables, use of sniffers dog by police or armed forces and use of farm dogs as shepherd [6].

Rearing of pet animals is day by day becoming popular in urban cities of Cameroon. A dog population of 695,001 was estimated in Cameroon composed of 320,534 owned dogs and 374,467 stray dogs [7]. Studies conducted in three regions of Cameroon suggested that over $66 \%$ households owned at least one animal in their compound and $46-76 \%$ households had at least one dog [8, 9]. As reported elsewhere, many Cameroonians are keeping dogs for protection and security of their houses and business premises, but like in many other African cultures, dogs are also kept as pets and companions, for hunting, as guarding animals, for commercial purposes, as well as a source of animal protein among some ethnic groups [8-12]. In Douala city for instance, they are kept mostly by households as pets, guard dog, as a source of commercial gains by breeders and by companies to assist in facility surveillance. Similar information on cat population is lacking. However, the pet owners do not have sufficient knowledge about the diseases, thus they are not aware of the diseases of pet animals, their medication and vaccination.

Although pet animals brings several benefits to our society, close interaction between humans and infected pets has been pointed out as an important risk factor for the incidence of many zoonotic diseases. Domestic carnivores' pathogens are helminths (31\%), bacteria (30\%), fungi (20\%), viruses (11\%), and protozoa (8\%), for a total of 374, out of which 261 (69.8\%) are known to also infected humans [13]. There are well-documented health hazards associated with owning a pet. Beside animal bites and allergy to pets, which are commonest health hazards, there is a diverse range of infections, including parasitic, bacterial, fungal and viral diseases that are capable of being transmitted to humans from domestic pets [2]. Other negative attributes of dogs such as noise, odor, waste pollution and free roaming nuisance affect the community's health at different levels of importance [10, 11]. Helminths such as Ancybstoma caninum, Strongyhides slenvralis, and Dipylidium caninum diagnosed in dogs of Dschang city are known to infect humans [14]. Some of infected pets contaminate water, soil and food that could be a leading source of infections in humans where they can produce mild to life threatening diseases [15]. Unfortunately, it have been reported that some zoonosis of major public health concern like helminths are ignored by a high proportion of pet owners $[8,12]$. Moreover, dog husbandry practices reported in the city of Yaounde in Cameroon, are likely to favor the spread of dog-related zoonotic diseases to humans [8].

In Cameroon, information concerning the epidemiology, diagnosis, treatment and management of pet diseases and those of zoonotic concern is largely scarce. Previous studies were focused on the prevalence of surgical pathologies and fight against surgical site infections in dogs and cats [16], canine 
rabies epidemiology $[7,17]$ and the prevalence of gastrointestinal helminths of dogs [14]. None of such reports focused on the profile of clinical diseases of pet dogs and cats. Hence, to reduce the gap in our knowledge of the distribution of clinically important diseases of pets, this retrospective study was undertaken to evaluate the prevalence of the most common clinical disease conditions of dogs and cats diagnosed in six private veterinary

clinics in Douala city, Cameroon. Likewise, determination of the main clinical signs and the effects of age, sex and breeds on the distribution of diseases. These data may be helpful for the veterinary practitioners as well as pet owners to take the necessary preventive measures to; control the diseases among dogs and cats, control of zoonotic diseases, guide disease diagnostic and preventative programs.

\section{Results}

For over five years (2014-2019), a total of 2397 cases of different clinical conditions were recorded in six private veterinary hospitals of Douala city in Cameroon, among them dogs and cats were 2222 (92.7\%) and 175 (7.3\%), respectively. Out of this number, 1448 animals were diseased, and 949 animals were apparently healthy representing $60.40 \%$ and $39.60 \%$, respectively. In dogs, diseases cases were more represented with a frequency of 1384 $(62.28 \%)$ as compare to surgical cases $(36,1.62 \%)$, vaccinations and health checkups $(802,36.09 \%)$. On the contrary, the clinical conditions most uncounted in cats were vaccinations and health checkups $(89,50.85 \%)$ as compare to diseases cases $(64,36.57 \%)$ and surgical cases $(22,12.57 \%)$.

Clinical signs registered in private veterinary practices (Fig. 1. and Table 1) 
Table 1

The 35 most common clinical signs and/or owners' complaints registered for dogs and cats

\begin{tabular}{|c|c|c|c|c|c|c|c|}
\hline \multirow{2}{*}{$\begin{array}{l}\text { Common Clinical signs/ } \\
\text { Owners' complaint }\end{array}$} & \multicolumn{2}{|c|}{ Cats, n (\%) } & \multirow{2}{*}{$\begin{array}{l}\text { Total Cats } \\
\mathrm{N}(\%)\end{array}$} & \multicolumn{2}{|l|}{ Dogs, n (\%) } & \multirow{2}{*}{$\begin{array}{l}\text { Total Dogs } \\
\mathrm{N}(\%)\end{array}$} & \multirow[t]{2}{*}{ Total } \\
\hline & Female & Male & & Female & Male & & \\
\hline Cardio-respiratory disorders & $0(0.0)$ & $0(0.0)$ & $0(0)$ & $9(0.6)$ & $20(1.4)$ & $29(2)$ & $29(2)$ \\
\hline Coughing & $0(0.0)$ & $0(0.0)$ & $0(0)$ & $4(0.3)$ & $7(0.5)$ & $11(0.8)$ & $11(0.8)$ \\
\hline Dyspnea & $0(0.0)$ & $0(0.0)$ & $0(0)$ & $2(0.1)$ & $5(0.3)$ & $7(0.5)$ & $7(0.5)$ \\
\hline Nasal discharge & $0(0.0)$ & $0(0.0)$ & $0(0)$ & $2(0.1)$ & $7(0.5)$ & $9(0.6)$ & $9(0.6)$ \\
\hline Tachycardia & $0(0.0)$ & $0(0.0)$ & $0(0)$ & $0(0)$ & $1(0.1)$ & $1(0.1)$ & $1(0.1)$ \\
\hline Tachypnea & $0(0.0)$ & $0(0.0)$ & $0(0)$ & $1(0.1)$ & $0(0)$ & $1(0.1)$ & $1(0.1)$ \\
\hline Digestive disorders & $10(0.7)$ & $16(1.1)$ & $26(1.8)$ & $277(19.1)$ & $541(37.4)$ & $818(56.5)$ & 844 (58.3) \\
\hline Abdominal bloating & $1(0.1)$ & $3(0.2)$ & $4(0.3)$ & $6(0.4)$ & $23(1.6)$ & $29(2)$ & $33(2.3)$ \\
\hline Abdominal pain & $0(0)$ & $(0)$ & $(0)$ & $4(0.3)$ & $13(0.9)$ & $17(1.2)$ & $17(1.2)$ \\
\hline Anorexia & $3(0.2)$ & $4(0.3)$ & $7(0.5)$ & $102(7)$ & $202(14)$ & $304(21)$ & 311 (21.5) \\
\hline Diarrhea & $3(0.2)$ & $1(0.1)$ & $4(0.3)$ & $84(5.8)$ & $160(11)$ & $244(16.9)$ & $248(17.1)$ \\
\hline Hypersalivation & $0(0)$ & $2(0.1)$ & $2(0.1)$ & $7(0.5)$ & $18(1.2)$ & $25(1.7)$ & $27(1.9)$ \\
\hline Rectal prolapse & $0(0)$ & $2(0.1)$ & $2(0.1)$ & $0(0)$ & $0(0)$ & $0(0)$ & $2(0.1)$ \\
\hline Vomiting & $3(0.2)$ & $4(0.3)$ & $7(0.5)$ & $74(5.1)$ & 125 (8.6) & 199 (13.7) & $206(14.2)$ \\
\hline Eye disorders & $0(0)$ & $0(0)$ & $0(0)$ & $2(0.1)$ & $7(0.5)$ & $9(0.6)$ & $9(0.6)$ \\
\hline Blurred vision & $0(0)$ & $0(0)$ & $0(0)$ & $2(0.1)$ & $7(0.5)$ & $9(0.6)$ & $9(0.6)$ \\
\hline General system disorders & $0(0)$ & $4(0.3)$ & $4(0.3)$ & $53(3.7)$ & $109(7.5)$ & $162(11.2)$ & $166(11.5)$ \\
\hline Anemia & $0(0)$ & $1(0.1)$ & $1(0.1)$ & $12(0.8)$ & $21(1.5)$ & $33(2.3)$ & $34(2.3)$ \\
\hline Cachexia & $0(0)$ & $1(0.1)$ & $1(0.1)$ & $3(0.2)$ & $15(1)$ & $18(1.2)$ & $19(1.3)$ \\
\hline Cyanosis & $0(0)$ & $0(0)$ & $0(0)$ & $0(0)$ & $4(0.3)$ & $4(0.3)$ & $4(0.3)$ \\
\hline Fever & $0(0)$ & $0(0)$ & $0(0)$ & $6(0.4)$ & $8(0.6)$ & $14(1)$ & $14(1)$ \\
\hline Ictera & $0(0)$ & $0(0)$ & $0(0)$ & $2(0.1)$ & $1(0.1)$ & $3(0.2)$ & $3(0.2)$ \\
\hline Spasm & $0(0)$ & $0(0)$ & $0(0)$ & $6(0.4)$ & $12(0.8)$ & $18(1.2)$ & $18(1.2)$ \\
\hline Tiredness & $0(0)$ & $2(0.1)$ & $2(0.1)$ & $24(1.7)$ & 48 (3.3) & $72(5)$ & $74(5.1)$ \\
\hline Genito-urinary disorders & $0(0)$ & $2(0.1)$ & $2(0.1)$ & $7(0.5)$ & $7(0.5)$ & $14(1)$ & $16(1.1)$ \\
\hline Hematuria & $0(0)$ & $0(0)$ & $0(0)$ & $4(0.3)$ & $4(0.3)$ & $8(0.6)$ & $8(0.6)$ \\
\hline Urination disorder & $0(0)$ & $2(0.1)$ & $2(0.1)$ & $0(0)$ & $3(0.2)$ & $3(0.2)$ & $5(0.3)$ \\
\hline Vaginal discharge & $0(0)$ & $0(0)$ & $0(0)$ & $3(0.2)$ & $0(0)$ & $3(0.2)$ & $3(0.2)$ \\
\hline Musculoskeletal disorders & $8(0.6)$ & $16(1.1)$ & $24(1.7)$ & $47(3.2)$ & $118(8.1)$ & $165(11.4)$ & $189(13.1)$ \\
\hline Accidental wounds & $0(0)$ & $3(0.2)$ & $3(0.2)$ & $7(0.5)$ & $18(1.2)$ & $25(1.7)$ & $28(1.9)$ \\
\hline Ataxia & $0(0)$ & $0(0)$ & $0(0)$ & $1(0.1)$ & $8(0.6)$ & $9(0.6)$ & $9(0.6)$ \\
\hline Hemorrhage & $1(0.1)$ & $0(0)$ & $1(0.1)$ & $1(0.1)$ & $1(0.1)$ & $2(0.1)$ & $3(0.2)$ \\
\hline Hematoma & $0(0)$ & $2(0.1)$ & $2(0.1)$ & $5(0.3)$ & $22(1.5)$ & $27(1.9)$ & $29(2)$ \\
\hline Inflammation & $3(0.2)$ & $4(0.3)$ & $7(0.5)$ & $11(0.8)$ & $33(2.3)$ & $44(3)$ & $51(3.5)$ \\
\hline Lameness & $4(0.3)$ & $2(0.1)$ & $6(0.4)$ & $12(0.8)$ & $26(1.8)$ & 38 (2.6) & $44(3)$ \\
\hline lymphadenopathy & $0(0)$ & $0(0)$ & $0(0)$ & $0(0)$ & $1(0.1)$ & $1(0.1)$ & $1(0.1)$ \\
\hline Paralysis & $0(0)$ & $2(0.1)$ & $2(0.1)$ & $1(0.1)$ & $3(0.2)$ & $4(0.3)$ & $6(0.4)$ \\
\hline Pus in antrum & $0(0)$ & $1(0.1)$ & $1(0.1)$ & $4(0.3)$ & $2(0.1)$ & $6(0.4)$ & $7(0.5)$ \\
\hline
\end{tabular}




\begin{tabular}{|c|c|c|c|c|c|c|c|}
\hline \multirow{2}{*}{$\begin{array}{l}\text { Common Clinical signs/ } \\
\text { Owners' complaint }\end{array}$} & \multicolumn{2}{|c|}{ Cats, n (\%) } & \multirow{2}{*}{$\begin{array}{l}\text { Total Cats } \\
\mathrm{N}(\%)\end{array}$} & \multicolumn{2}{|l|}{ Dogs, n (\%) } & \multirow{2}{*}{$\begin{array}{l}\text { Total Dogs } \\
\text { N (\%) }\end{array}$} & \multirow[t]{2}{*}{ Total } \\
\hline & Female & Male & & Female & Male & & \\
\hline Swelling & $0(0)$ & $2(0.1)$ & $2(0.1)$ & $5(0.3)$ & $4(0.3)$ & $9(0.6)$ & $11(0.8)$ \\
\hline Skin disorders & $2(0.1)$ & $6(0.4)$ & $8(0.6)$ & $52(3.6)$ & $135(9.3)$ & $187(12.9)$ & 195 (13.5) \\
\hline Ectoparasites & $0(0)$ & $0(0)$ & $0(0)$ & $25(1.7)$ & $51(3.5)$ & $76(5.2)$ & $76(5.2)$ \\
\hline Pruritis & $2(0.1)$ & $6(0.4)$ & $8(0.6)$ & $27(1.9)$ & $84(5.8)$ & $111(7.7)$ & $119(8.2)$ \\
\hline Overall frequency & $20(1.4)$ & 44 (3)* & $64(4.4)$ & $447(30.9)$ & 937 (64.7)* & $1384(95.6)$ & $1448(100)$ \\
\hline
\end{tabular}

A total of 35 common clinical symptoms and/or owners' complaints were recorded in dogs and cats and classified according to organ system affected. Digestive disorders were found to account significantly $(P<0.0001)$ for $58.3 \%$ of the diagnosed cases, followed by skin disorders $(13.5 \%)$,

musculoskeletal disorders (13.1\%), general system disorders (11.5\%), cardio-respiratory disorders (2\%), genito-urinary disorders (1.1\%) and eye disorders (0.6\%, Fig. 1 and Table 1). This indicates that digestive disorders represented the major cause of clinical consultations in both owned dogs and cats with anorexia (21.5\%), diarrhea (17.1\%), vomiting (14.2\%), abdominal bloating (2.3\%), hypersalivation (1.9\%), abdominal pain (1.2\%) and rectal prolapse (0.1) ranking as the most clinical signs. Similarly, pruritis (8.2\%) and ectoparasites (5.2\%) were the most common skin disorders, while inflammation (3.5\%), lameness (3.0\%) and accidental wounds (1.9\%) were the gravest musculoskeletal disorders affecting the dogs and cats. Details on the others clinical signs and related organ systems are given.

Overall disease prevalence in dogs and cats and their sex-wise disease prevalence (Table 2 and Fig. 2) 
Table 2

Prevalence of common clinical diseases in dogs and cats and their distribution with relation to sex

\begin{tabular}{|c|c|c|c|c|c|c|c|c|}
\hline \multirow[t]{2}{*}{ Clinical diseases } & \multicolumn{3}{|l|}{ Dogs, n (\%) } & \multirow[t]{2}{*}{ P-value } & \multicolumn{3}{|l|}{ Cats, n (\%) } & \multirow[t]{2}{*}{ P-value } \\
\hline & $\begin{array}{l}\text { Number of } \\
\text { dogs }\end{array}$ & Female & Male & & $\begin{array}{l}\text { Number of } \\
\text { cats }\end{array}$ & Female & Male & \\
\hline Bacterial/fungal diseases & $30(1.4)$ & $15(0.7)$ & $15(0.7)$ & $X^{2}=4.5$ & $0(0)$ & $0(0)$ & $0(0)$ & \\
\hline Erlichiosis & $5(0.2)$ & $2(0.1)$ & $3(0.1)$ & $P=0.208$ & $0(0)$ & $0(0)$ & $0(0)$ & \\
\hline Leptospirosis & $7(0.3)$ & $5(0.2)$ & $2(0.1)$ & & $0(0)$ & $0(0)$ & $0(0)$ & I \\
\hline Ringworm & $3(0.1)$ & $0(0)$ & $3(0.1)$ & & $0(0)$ & $0(0)$ & $0(0)$ & \\
\hline Septicemia & $15(0.7)$ & $8(0.4)$ & $7(0.3)$ & & $0(0)$ & $0(0)$ & $0(0)$ & \\
\hline Non specific diseases & $558(25.1)$ & $153(6.9)$ & $\begin{array}{l}405 \\
(18.2)\end{array}$ & $X^{2}=65.5$ & $34(19.4)$ & $11(6.3)$ & $23(13.1)$ & $X^{2}=9.6$ \\
\hline Abscess & $6(0.3)$ & $4(0.2)$ & $2(0.1)$ & $\begin{array}{l}\mathrm{P}< \\
0.0001 *\end{array}$ & $1(0.6)$ & $0(0)$ & $1(0.6)$ & $P=0.474$ \\
\hline Ascites & $5(0.2)$ & $0(0)$ & $5(0.2)$ & & $0(0)$ & $0(0)$ & $0(0)$ & \\
\hline Aural haematoma & $20(0.9)$ & $2(0.1)$ & $18(0.8)$ & & $2(1.1)$ & $0(0)$ & $2(1.1)$ & \\
\hline Bronchitis & $2(0.1)$ & $1(0)$ & $1(0)$ & & $0(0)$ & $0(0)$ & $0(0)$ & \\
\hline Cataract & $3(0.1)$ & $1(0)$ & $2(0.1)$ & & $0(0)$ & $0(0)$ & $0(0)$ & \\
\hline Cherry eye & $1(0)$ & $0(0)$ & $1(0)$ & & $0(0)$ & $0(0)$ & $0(0)$ & \\
\hline Conjunctivitis & $5(0.2)$ & $1(0)$ & $4(0.2)$ & & $0(0)$ & $0(0)$ & $0(0)$ & \\
\hline Constipation & $5(0.2)$ & $0(0)$ & $5(0.2)$ & & $0(0)$ & $0(0)$ & $0(0)$ & \\
\hline Dermatitis & $63(2.8)$ & $17(0.8)$ & $46(2.1)$ & & $5(2.9)$ & $1(0.6)$ & $4(2.3)$ & \\
\hline Eczema & $19(0.9)$ & $5(0.2)$ & $14(0.6)$ & & $2(1,1)$ & $1(0.6)$ & $1(0.6)$ & \\
\hline Edema & $3(0.1)$ & $1(0)$ & $2(0.1)$ & & $0(0)$ & $0(0)$ & $0(0)$ & \\
\hline Epileptiform crisis & $2(0.1)$ & $2(0.1)$ & $0(0)$ & & $0(0)$ & $0(0)$ & $0(0)$ & \\
\hline Epistasis & $1(0)$ & $0(0)$ & $1(0)$ & & $1(0.6)$ & $1(0.6)$ & $0(0)$ & \\
\hline Gastroenteritis & $174(7.8)$ & $52(2.3)$ & $122(5.5)$ & & $2(1.1)$ & $2(1.1)$ & $0(0)$ & \\
\hline Haemorrhage & $1(0)$ & $1(0)$ & $0(0)$ & & $0(0)$ & $0(0)$ & $0(0)$ & \\
\hline Hematoma & $7(0.3)$ & $3(0.1)$ & $4(0.2)$ & & $0(0)$ & $0(0)$ & $0(0)$ & \\
\hline Mastitis & $3(0.1)$ & $3(0.1)$ & $0(0)$ & & $0(0)$ & $0(0)$ & $0(0)$ & \\
\hline Metritis/pyometra & $3(0.1)$ & $3(0.1)$ & $0(0)$ & & $0(0)$ & $0(0)$ & $0(0)$ & \\
\hline Orchitis & $10(0.5)$ & $0(0)$ & $10(0.5)$ & & $0(0)$ & $0(0)$ & $0(0)$ & \\
\hline Otitis & $25(1.1)$ & $3(0.1)$ & $22(1)$ & & $2(1.1)$ & $0(0)$ & $2(1.1)$ & \\
\hline Paralysis & $4(0.2)$ & $1(0)$ & $3(0.1)$ & & $2(1.1)$ & $0(0)$ & $2(1.1)$ & \\
\hline Paraphimosis & $2(0.1)$ & $0(0)$ & $2(0.1)$ & & $0(0)$ & $0(0)$ & $0(0)$ & \\
\hline Pneumonia & $5(0.2)$ & $0(0)$ & $5(0.2)$ & & $0(0)$ & $0(0)$ & $0(0)$ & \\
\hline Poisoning & $174(7.8)$ & $44(2)$ & $130(5.9)$ & & $9(5.1)$ & $4(2.3)$ & $5(2.9)$ & \\
\hline Rectal prolapse & $0(0)$ & $0(0)$ & $0(0)$ & & $2(1.1)$ & $0(0)$ & $2(1.1)$ & \\
\hline Tumor & $9(0.4)$ & $6(0.3)$ & $3(0.1)$ & & $4(2.3)$ & $2(1.1)$ & $2(1.1)$ & \\
\hline Umbilical hernia & $3(0.1)$ & $3(0.1)$ & $0(0)$ & & $0(0)$ & $0(0)$ & $0(0)$ & \\
\hline Urinary infection & $3(0.1)$ & $0(0)$ & $3(0.1)$ & & $2(1.1)$ & $0(0)$ & $2(1.1)$ & \\
\hline Non-infectious diseases & $81(3.6)$ & $24(1.1)$ & $57(2.6)$ & $X^{2}=3.1$ & $13(7.4)$ & $5(2.9)$ & $8(4.6)$ & $X^{2}=3.7$ \\
\hline Accidental injury & $24(1.1)$ & $10(0.5)$ & $14(0.6)$ & $P=0.676$ & $5(2.9)$ & $3(1.7)$ & $2(1.1)$ & $P=0.295$ \\
\hline
\end{tabular}

$\mathrm{X}^{2}=$ Chi-squared value; $\left.{ }^{*}\right)$ indicate a significant association 


\begin{tabular}{|c|c|c|c|c|c|c|c|c|}
\hline \multirow[t]{2}{*}{ Clinical diseases } & \multicolumn{3}{|l|}{ Dogs, n (\%) } & \multirow[t]{2}{*}{ P-value } & \multicolumn{3}{|l|}{ Cats, n (\%) } & \multirow[t]{2}{*}{ P-value } \\
\hline & $\begin{array}{l}\text { Number of } \\
\text { dogs }\end{array}$ & Female & Male & & $\begin{array}{l}\text { Number of } \\
\text { cats }\end{array}$ & Female & Male & \\
\hline Dislocation & $3(0.1)$ & $1(0)$ & $2(0.1)$ & & $0(0)$ & $0(0)$ & $0(0)$ & \\
\hline food hypersensitivity & $12(0.5)$ & $2(0.1)$ & $10(0.5)$ & & $1(0.6)$ & $0(0)$ & $1(0.6)$ & \\
\hline Fracture & $14(0.6)$ & $3(0.1)$ & $11(0.5)$ & & $4(2.3)$ & $2(1.1)$ & $2(1.1)$ & \\
\hline Sprain & $3(0.1)$ & $1(0)$ & $2(0.1)$ & & $0(0)$ & $0(0)$ & $0(0)$ & \\
\hline Wounds & $25(1.1)$ & $7(0.3)$ & $18(0.8)$ & & $3(1.7)$ & $0(0)$ & $3(1.7)$ & \\
\hline Parasitic diseases & $283(12.7)$ & $93(4.2)$ & $190(8.6)$ & $X^{2}=1.6$ & $17(9.7)$ & $4(2.3)$ & $13(7.4)$ & \\
\hline Helminthoses & $183(8.2)$ & $63(2.8)$ & $120(5.4)$ & $P=0.641$ & $17(9.7)$ & $4(2.3)$ & $13(7.4)$ & \\
\hline Mites (Mange) & $15(0.7)$ & $3(0.1)$ & $12(0.5)$ & & $0(0)$ & $0(0)$ & $0(0)$ & I \\
\hline Myiasis & $32(1.4)$ & $9(0.4)$ & $23(1)$ & & $0(0)$ & $0(0)$ & $0(0)$ & \\
\hline Tick/Flea infestation & $53(2.4)$ & $18(0.8)$ & $35(1.6)$ & & $0(0)$ & $0(0)$ & $0(0)$ & \\
\hline Protozoan diseases & $59(2.7)$ & $25(1.1)$ & $34(1.5)$ & $X^{2}=17.9$ & $0(0)$ & $0(0)$ & $0(0)$ & \\
\hline Amoebiasis & $33(1.5)$ & $6(0.3)$ & $27(1.2)$ & $\begin{array}{l}\mathrm{P}< \\
0.0001^{*}\end{array}$ & $0(0)$ & $0(0)$ & $0(0)$ & I \\
\hline Babesiosis & $26(1.2)$ & $19(0.9)$ & $7(0.3)$ & & $0(0)$ & $0(0)$ & $0(0)$ & \\
\hline Viral diseases & $373(16.8)$ & $137(6.2)$ & $\begin{array}{l}236 \\
(10.6)\end{array}$ & $X^{2}=0.03$ & $0(0)$ & $0(0)$ & $0(0)$ & \\
\hline Kennel cough & $18(0.8)$ & $7(0.3)$ & $11(0.5)$ & $P=0.846$ & $0(0)$ & $0(0)$ & $0(0)$ & I \\
\hline Parvovirosis & $355(16)$ & $130(5.9)$ & $\begin{array}{l}225 \\
(10.1)\end{array}$ & & $0(0)$ & $0(0)$ & $0(0)$ & \\
\hline Convenience surgery & $36(1.6)$ & $13(0.6)$ & $23(1)$ & $X^{2}=19.2$ & $22(12.6)$ & $8(4.6)$ & $14(8)$ & $X^{2}=22.0$ \\
\hline Castration & $16(0.7)$ & $1(0)$ & $15(0.7)$ & $P=0.001 *$ & $14(8)$ & $0(0)$ & $14(8)$ & $\begin{array}{l}\mathrm{P}< \\
0.0001^{*}\end{array}$ \\
\hline Otectomy & $1(0)$ & $0(0)$ & $1(0)$ & & $0(0)$ & $0(0)$ & $0(0)$ & \\
\hline Ovariectomy & $2(0.1)$ & $2(0.1)$ & $0(0)$ & & $6(3.4)$ & $6(3.4)$ & $0(0)$ & \\
\hline Ovariohysterectomy & $5(0.2)$ & $5(0.2)$ & $0(0)$ & & $2(1.1)$ & $2(1.1)$ & $0(0)$ & \\
\hline Tail docking & $12(0.5)$ & $5(0.2)$ & $7(0.3)$ & & $0(0)$ & $0(0)$ & $0(0)$ & \\
\hline $\begin{array}{l}\text { Vaccination \& health check } \\
\text { up }\end{array}$ & $802(36.1)$ & $\begin{array}{l}273 \\
(12.3)\end{array}$ & $\begin{array}{l}529 \\
(23.8)\end{array}$ & I & $89(50.9)$ & $\begin{array}{l}43 \\
(24.6)\end{array}$ & $46(26.3)$ & $89(3.7)$ \\
\hline Overall prevalence & $2222(100)$ & $733(33)$ & $1489(67)$ & I & $175(100)$ & $\begin{array}{l}71 \\
(40.6)\end{array}$ & $\begin{array}{l}104 \\
(59.4)\end{array}$ & $175(7.3)$ \\
\hline
\end{tabular}

A total of 46 types of clinical diseases in six categories were recorded in pet dogs and cats. Amongst these diseases the high prevalence were reported in nonspecific clinical diseases (dogs $25.1 \%$ and cats $19.4 \%$ ) followed by viral diseases (dogs $16.8 \%$ ), parasitic diseases (dogs $12.7 \%$ and cats $9.7 \%$ ), protozoan diseases (dogs $2.7 \%$ ) and bacterial/fungal diseases (dogs 1.4\%). When grouped together, infectious diseases appears to be most high in dogs than cats with respective prevalence of $33.6 \%$ and $9.7 \%$. For dogs, the most occurring clinical diseases comprised parvovirosis (16\%), helminthoses ( $8.2 \%)$, gastroenteritis (7.8\%), poisoning (7.8\%), dermatitis (2.8\%), babesiosis (1.2\%), amoebiasis (1.5\%), otitis (1.1\%), accidental injury (1.1\%) and wounds (1.1\%). Overall prevalence of clinical diseases of pet cats showed highest prevalence of helminthoses (9.7\%) followed by poisoning (5.1\%), dermatitis (2.9\%), accidental injury (2.9\%), fracture (2.3\%), tumor (2.3\%) and wounds (1.7\%). The sex-wise highest prevalence of diseases in both dogs and cats was observed in male (dogs $67 \%$ and cats $59.4 \%$ ) than female (dogs $33 \%$ and cats $40.6 \%$ ) with a significant difference $(p=0.009$, Fig. 2 ). Male dogs were found to be more affected with aural haematoma, dermatitis, eczema, gastroenteritis, orchitis, poisoning, wounds, amoebiasis, while females were more prone to abscess, tumor, umbilical hernia and babesiosis $(p<0.0001$, Table 2$)$.

Effects of breed on the cases diagnosed (Table 3 and Fig. 3) 
Table 3

Association between major clinical diseases of dogs and cats with breed

\begin{tabular}{|c|c|c|c|c|c|c|c|c|c|c|c|c|}
\hline \multirow[t]{2}{*}{ Clinical diseases } & \multicolumn{9}{|l|}{ Dogs } & \multicolumn{3}{|l|}{ Cats } \\
\hline & $\begin{array}{l}\text { Cross } \\
\text { (Mixed) }\end{array}$ & $\begin{array}{l}\text { Local } \\
\text { breed }\end{array}$ & $\begin{array}{l}\text { German } \\
\text { Shepherd }\end{array}$ & $\begin{array}{l}\text { Toy } \\
\text { breeds } \\
t\end{array}$ & Rottweiler & $\begin{array}{l}\text { Cane } \\
\text { corso }\end{array}$ & $\begin{array}{l}\text { Great } \\
\text { Dane }\end{array}$ & $\begin{array}{l}\text { Other } \\
\text { exotics } \\
\neq\end{array}$ & p-value & $\begin{array}{l}\text { Exotic } \\
\text { Cat }\end{array}$ & $\begin{array}{l}\text { Local } \\
\text { Cat }\end{array}$ & $\begin{array}{l}\text { P- } \\
\text { Value }\end{array}$ \\
\hline $\begin{array}{l}\text { Bacterial/fungal } \\
\text { diseases }\end{array}$ & $\begin{array}{l}11 \\
(0.5)\end{array}$ & $\begin{array}{l}6 \\
(0.3)\end{array}$ & $8(0.4)$ & $3(0.1)$ & $2(0.1)$ & $0(0)$ & $0(0)$ & $0(0)$ & $X^{2}=45.1$ & $0(0)$ & $0(0)$ & I \\
\hline Erlichiosis & $0(0)$ & $0(0)$ & $0(0)$ & $3(0.1)$ & $2(0.1)$ & $0(0)$ & $0(0)$ & $0(0)$ & $\begin{array}{l}P< \\
0.0001 *\end{array}$ & $0(0)$ & $0(0)$ & \\
\hline Leptospirosis & $7(0.3)$ & $0(0)$ & $0(0)$ & $0(0)$ & $0(0)$ & $0(0)$ & $0(0)$ & $0(0)$ & & $0(0)$ & $0(0)$ & \\
\hline Ringworm & $1(0)$ & $1(0)$ & $1(0)$ & $0(0)$ & $0(0)$ & $0(0)$ & $0(0)$ & $0(0)$ & & $0(0)$ & $0(0)$ & \\
\hline Septicemia & $3(0.1)$ & $\begin{array}{l}5 \\
(0.2)\end{array}$ & $7(0.3)$ & $0(0)$ & $0(0)$ & $0(0)$ & $0(0)$ & $0(0)$ & & $0(0)$ & $0(0)$ & \\
\hline $\begin{array}{l}\text { Non specific } \\
\text { diseases }\end{array}$ & $\begin{array}{l}198 \\
(8.9)\end{array}$ & $\begin{array}{l}96 \\
(4.3)\end{array}$ & $84(3.8)$ & $\begin{array}{l}37 \\
(1.7)\end{array}$ & $28(1.3)$ & $\begin{array}{l}21 \\
(0.9)\end{array}$ & $\begin{array}{l}23 \\
(1)\end{array}$ & $\begin{array}{l}71 \\
(3.2)\end{array}$ & $X^{2}=454.7$ & $\begin{array}{l}3 \\
(1.7)\end{array}$ & $\begin{array}{l}31 \\
(17.7)\end{array}$ & $X^{2}=15.3$ \\
\hline Abscess & $1(0)$ & $\begin{array}{l}2 \\
(0.1)\end{array}$ & $0(0)$ & $0(0)$ & $0(0)$ & $0(0)$ & $0(0)$ & $3(0.1)$ & $\begin{array}{l}\mathrm{P}< \\
0.0001 *\end{array}$ & $0(0)$ & $\begin{array}{l}1 \\
(0.6)\end{array}$ & $\begin{array}{l}P= \\
0.167\end{array}$ \\
\hline Ascites & $1(0)$ & $\begin{array}{l}2 \\
(0.1)\end{array}$ & $2(0.1)$ & $0(0)$ & $0(0)$ & $0(0)$ & $0(0)$ & $0(0)$ & & $0(0)$ & $0(0)$ & \\
\hline Aural haematoma & $2(0.1)$ & $1(0)$ & $9(0.4)$ & $1(0)$ & $0(0)$ & $\begin{array}{l}7 \\
(0.3)\end{array}$ & $0(0)$ & $0(0)$ & & $0(0)$ & $\begin{array}{l}2 \\
(1.1)\end{array}$ & \\
\hline Bronchitis & $0(0)$ & $1(0)$ & $0(0)$ & $0(0)$ & $1(0)$ & $0(0)$ & $0(0)$ & $0(0)$ & & $0(0)$ & $0(0)$ & \\
\hline Cataract & $3(0.1)$ & $0(0)$ & $0(0)$ & $0(0)$ & $0(0)$ & $0(0)$ & $0(0)$ & $0(0)$ & & $0(0)$ & $0(0)$ & \\
\hline Cherry eye & $1(0)$ & $0(0)$ & $0(0)$ & $0(0)$ & $0(0)$ & $0(0)$ & $0(0)$ & $0(0)$ & & $0(0)$ & $0(0)$ & \\
\hline Conjunctivitis & $2(0.1)$ & $1(0)$ & $0(0)$ & $2(0.1)$ & $0(0)$ & $0(0)$ & $0(0)$ & $0(0)$ & & $0(0)$ & $0(0)$ & \\
\hline Constipation & $2(0.1)$ & $\begin{array}{l}3 \\
(0.1)\end{array}$ & $0(0)$ & $0(0)$ & $0(0)$ & $0(0)$ & $0(0)$ & $0(0)$ & & $0(0)$ & $0(0)$ & \\
\hline Dermatitis & $\begin{array}{l}17 \\
(0.8)\end{array}$ & $\begin{array}{l}10 \\
(0.5)\end{array}$ & $8(0.4)$ & $2(0.1)$ & $8(0.4)$ & $\begin{array}{l}4 \\
(0.2)\end{array}$ & $\begin{array}{l}3 \\
(0.1)\end{array}$ & $\begin{array}{l}11 \\
(0.5)\end{array}$ & & $0(0)$ & $\begin{array}{l}5 \\
(2.9)\end{array}$ & \\
\hline Eczema & $1(0)$ & $1(0)$ & $10(0.5)$ & $0(0)$ & $1(0)$ & $1(0)$ & $1(0)$ & $4(0.2)$ & & $0(0)$ & $\begin{array}{l}2 \\
(1.1)\end{array}$ & \\
\hline Edema & $1(0)$ & $0(0)$ & $0(0)$ & $0(0)$ & $0(0)$ & $0(0)$ & $0(0)$ & $2(0.1)$ & & $0(0)$ & $0(0)$ & \\
\hline Epileptiform crisis & $0(0)$ & $0(0)$ & $0(0)$ & $2(0.1)$ & $0(0)$ & $0(0)$ & $0(0)$ & $0(0)$ & & $0(0)$ & $0(0)$ & \\
\hline Epistasis & $1(0)$ & $0(0)$ & $0(0)$ & $0(0)$ & $0(0)$ & $0(0)$ & $0(0)$ & $0(0)$ & & $0(0)$ & $\begin{array}{l}1 \\
(0.6)\end{array}$ & \\
\hline Gastroenteritis & $\begin{array}{l}101 \\
(4.5)\end{array}$ & $\begin{array}{l}21 \\
(0.9)\end{array}$ & $9(0.4)$ & $\begin{array}{l}12 \\
(0.5)\end{array}$ & $5(0.2)$ & $\begin{array}{l}4 \\
(0.2)\end{array}$ & $\begin{array}{l}6 \\
(0.3)\end{array}$ & $\begin{array}{l}16 \\
(0.7)\end{array}$ & & $0(0)$ & $\begin{array}{l}2 \\
(1.1)\end{array}$ & \\
\hline Haemorrhage & $0(0)$ & $0(0)$ & $0(0)$ & $0(0)$ & $0(0)$ & $1(0)$ & $0(0)$ & $0(0)$ & & $0(0)$ & $0(0)$ & \\
\hline Hematoma & $1(0)$ & $\begin{array}{l}3 \\
(0.1)\end{array}$ & $0(0)$ & $1(0)$ & $0(0)$ & $1(0)$ & $0(0)$ & $1(0)$ & & $0(0)$ & $0(0)$ & \\
\hline Mastitis & $0(0)$ & $1(0)$ & $1(0)$ & $0(0)$ & $0(0)$ & $0(0)$ & $0(0)$ & $1(0)$ & & $0(0)$ & $0(0)$ & \\
\hline Metritis/pyometra & $3(0.1)$ & $0(0)$ & $0(0)$ & $0(0)$ & $0(0)$ & $0(0)$ & $0(0)$ & $0(0)$ & & $0(0)$ & $0(0)$ & \\
\hline Orchitis & $3(0.1)$ & $\begin{array}{l}2 \\
(0.1)\end{array}$ & $5(0.2)$ & $0(0)$ & $0(0)$ & $0(0)$ & $0(0)$ & $0(0)$ & & $0(0)$ & $0(0)$ & \\
\hline Otitis & $5(0.2)$ & $1(0)$ & $13(0.6)$ & $4(0.2)$ & $1(0)$ & $0(0)$ & $0(0)$ & $1(0)$ & & $\begin{array}{l}1 \\
(0.6)\end{array}$ & $\begin{array}{l}1 \\
(0.6)\end{array}$ & \\
\hline Paralysis & $0(0)$ & $\begin{array}{l}4 \\
(0.2)\end{array}$ & $0(0)$ & $0(0)$ & $0(0)$ & $0(0)$ & $0(0)$ & $0(0)$ & & $0(0)$ & $\begin{array}{l}2 \\
(1.1)\end{array}$ & \\
\hline
\end{tabular}

$\mathrm{X}^{2}=$ Chi-squared value; $\left({ }^{\star}\right)$ indicate a significant association; $(\dagger)$ Toy breeds were represented by Shih Tzu, Chihuahua, Bichon, Cavalier King Charles, Cotton tulear and poodle; ( $¥)$ Other exotics were represented by Doberman, Boerboel, Labrador, Bulldog, Belgian Shepherd, Dalmatian, Caucasian and Samoyed. 


\begin{tabular}{|c|c|c|c|c|c|c|c|c|c|c|c|c|}
\hline \multirow[t]{2}{*}{ Clinical diseases } & \multicolumn{9}{|l|}{ Dogs } & \multicolumn{3}{|l|}{ Cats } \\
\hline & $\begin{array}{l}\text { Cross } \\
\text { (Mixed) }\end{array}$ & $\begin{array}{l}\text { Local } \\
\text { breed }\end{array}$ & $\begin{array}{l}\text { German } \\
\text { Shepherd }\end{array}$ & $\begin{array}{l}\text { Toy } \\
\text { breeds } \\
t\end{array}$ & Rottweiler & $\begin{array}{l}\text { Cane } \\
\text { corso }\end{array}$ & $\begin{array}{l}\text { Great } \\
\text { Dane }\end{array}$ & $\begin{array}{l}\text { Other } \\
\text { exotics } \\
\ddagger\end{array}$ & p-value & $\begin{array}{l}\text { Exotic } \\
\text { Cat }\end{array}$ & $\begin{array}{l}\text { Local } \\
\text { Cat }\end{array}$ & $\begin{array}{l}\text { P- } \\
\text { Value }\end{array}$ \\
\hline Paraphimosis & $0(0)$ & $0(0)$ & $0(0)$ & $0(0)$ & $0(0)$ & $0(0)$ & $1(0)$ & $1(0)$ & & $0(0)$ & $0(0)$ & \\
\hline Pneumonia & $0(0)$ & $0(0)$ & $1(0)$ & $0(0)$ & $3(0.1)$ & $0(0)$ & $0(0)$ & $1(0)$ & & $0(0)$ & $0(0)$ & \\
\hline Poisoning & $\begin{array}{l}50 \\
(2.3)\end{array}$ & $\begin{array}{l}38 \\
(1.7)\end{array}$ & $25(1.1)$ & $\begin{array}{l}13 \\
(0.6)\end{array}$ & $8(0.4)$ & $\begin{array}{l}3 \\
(0.1)\end{array}$ & $\begin{array}{l}12 \\
(0.5)\end{array}$ & $\begin{array}{l}25 \\
(1.1)\end{array}$ & & $0(0)$ & $\begin{array}{l}9 \\
(5.1)\end{array}$ & \\
\hline Rectal prolapse & $0(0)$ & $0(0)$ & $0(0)$ & $0(0)$ & $0(0)$ & $0(0)$ & $0(0)$ & $0(0)$ & & $0(0)$ & $\begin{array}{l}2 \\
(1.1)\end{array}$ & \\
\hline Tumor & $0(0)$ & $\begin{array}{l}2 \\
(0.1)\end{array}$ & $1(0)$ & $0(0)$ & $1(0)$ & $0(0)$ & $0(0)$ & $5(0.2)$ & & $\begin{array}{l}2 \\
(1.1)\end{array}$ & $\begin{array}{l}2 \\
(1.1)\end{array}$ & \\
\hline Umbilical hernia & $0(0)$ & $\begin{array}{l}3 \\
(0.1)\end{array}$ & $0(0)$ & $0(0)$ & $0(0)$ & $0(0)$ & $0(0)$ & $0(0)$ & & $0(0)$ & $0(0)$ & \\
\hline Urinary infection & $3(0.1)$ & $0(0)$ & $0(0)$ & $0(0)$ & $0(0)$ & $0(0)$ & $0(0)$ & $0(0)$ & & $0(0)$ & $\begin{array}{l}2 \\
(1.1)\end{array}$ & \\
\hline $\begin{array}{l}\text { Non-infectious } \\
\text { diseases }\end{array}$ & $\begin{array}{l}14 \\
(0.6)\end{array}$ & $\begin{array}{l}24 \\
(1.1)\end{array}$ & $9(0.4)$ & $\begin{array}{l}12 \\
(0.5)\end{array}$ & $4(0.2)$ & $\begin{array}{l}5 \\
(0.2)\end{array}$ & $1(0)$ & $\begin{array}{l}12 \\
(0.5)\end{array}$ & $X^{2}=41.8$ & $\begin{array}{l}3 \\
(1.7)\end{array}$ & $\begin{array}{l}10 \\
(5.7)\end{array}$ & $X^{2}=2.4$ \\
\hline Accidental injury & $6(0.3)$ & $\begin{array}{l}8 \\
(0.4)\end{array}$ & $3(0.1)$ & $3(0.1)$ & $1(0)$ & $1(0)$ & $0(0)$ & $2(0.1)$ & $P=0.197$ & $\begin{array}{l}2 \\
(1.1)\end{array}$ & $\begin{array}{l}3 \\
(1.7)\end{array}$ & $\begin{array}{l}P= \\
0.478\end{array}$ \\
\hline Dislocation & $0(0)$ & $1(0)$ & $0(0)$ & $2(0.1)$ & $0(0)$ & $0(0)$ & $0(0)$ & $0(0)$ & & $0(0)$ & $0(0)$ & \\
\hline $\begin{array}{l}\text { food } \\
\text { hypersensitivity }\end{array}$ & $1(0)$ & $1(0)$ & $0(0)$ & $3(0.1)$ & $0(0)$ & $\begin{array}{l}2 \\
(0.1)\end{array}$ & $0(0)$ & $5(0.2)$ & & $0(0)$ & $\begin{array}{l}1 \\
(0.6)\end{array}$ & \\
\hline Fracture & $2(0.1)$ & $\begin{array}{l}7 \\
(0.3)\end{array}$ & $3(0.1)$ & $1(0)$ & $0(0)$ & $0(0)$ & $0(0)$ & $1(0)$ & & $0(0)$ & $\begin{array}{l}4 \\
(2.3)\end{array}$ & \\
\hline Sprain & $0(0)$ & $1(0)$ & $0(0)$ & $0(0)$ & $1(0)$ & $1(0)$ & $0(0)$ & $0(0)$ & & $0(0)$ & $0(0)$ & \\
\hline Wounds & $5(0.2)$ & $\begin{array}{l}6 \\
(0.3)\end{array}$ & $3(0.1)$ & $3(0.1)$ & $2(0.1)$ & $1(0)$ & $1(0)$ & $4(0.2)$ & & $\begin{array}{l}1 \\
(0.6)\end{array}$ & $\begin{array}{l}2 \\
(1.1)\end{array}$ & \\
\hline Parasitic diseases & $\begin{array}{l}90 \\
(4.1)\end{array}$ & $\begin{array}{l}102 \\
(4.6)\end{array}$ & $31(1.4)$ & $\begin{array}{l}26 \\
(1.2)\end{array}$ & $11(0.5)$ & $\begin{array}{l}4 \\
(0.2)\end{array}$ & $\begin{array}{l}2 \\
(0.1)\end{array}$ & $\begin{array}{l}17 \\
(0.8)\end{array}$ & $X^{2}=45.6$ & $\begin{array}{l}1 \\
(0.6)\end{array}$ & $\begin{array}{l}16 \\
(9.1)\end{array}$ & \\
\hline Helminthoses & $\begin{array}{l}65 \\
(2.9)\end{array}$ & $\begin{array}{l}64 \\
(2.9)\end{array}$ & $21(0.9)$ & $\begin{array}{l}12 \\
(0.5)\end{array}$ & $7(0.3)$ & $\begin{array}{l}3 \\
(0.1)\end{array}$ & $\begin{array}{l}2 \\
(0.1)\end{array}$ & $9(0.4)$ & $\begin{array}{l}\mathrm{P}= \\
0.001 *\end{array}$ & $\begin{array}{l}1 \\
(0.6)\end{array}$ & $\begin{array}{l}16 \\
(9.1)\end{array}$ & \\
\hline Mites (Mange) & $2(0.1)$ & $\begin{array}{l}2 \\
(0.1)\end{array}$ & $5(0.2)$ & $1(0)$ & $1(0)$ & $0(0)$ & $0(0)$ & $4(0.2)$ & & $0(0)$ & $0(0)$ & l \\
\hline Myiasis & $5(0.2)$ & $\begin{array}{l}19 \\
(0.9)\end{array}$ & $0(0)$ & $7(0.3)$ & $0(0)$ & $0(0)$ & $0(0)$ & $1(0)$ & & $0(0)$ & $0(0)$ & \\
\hline $\begin{array}{l}\text { Tick/Flea } \\
\text { infestation }\end{array}$ & $\begin{array}{l}18 \\
(0.8)\end{array}$ & $\begin{array}{l}17 \\
(0.8)\end{array}$ & $5(0.2)$ & $6(0.3)$ & $3(0.1)$ & $1(0)$ & $0(0)$ & $3(0.1)$ & & $0(0)$ & $0(0)$ & \\
\hline Protozoan diseases & $\begin{array}{l}33(1 . \\
5)\end{array}$ & $\begin{array}{l}14 \\
(0.6)\end{array}$ & $10(0.5)$ & $0(0)$ & $1(0)$ & $0(0)$ & $0(0)$ & $1(0)$ & $X^{2}=12.09$ & $0(0)$ & $0(0)$ & \\
\hline Amoebiasis & $\begin{array}{l}14 \\
(0.6)\end{array}$ & $\begin{array}{l}7 \\
(0.3)\end{array}$ & $10(0.5)$ & $0(0)$ & $1(0)$ & $0(0)$ & $0(0)$ & $1(0)$ & $\begin{array}{l}P= \\
0.017 *\end{array}$ & $0(0)$ & $0(0)$ & / \\
\hline Babesiosis & $\begin{array}{l}19 \\
(0.9)\end{array}$ & $\begin{array}{l}7 \\
(0.3)\end{array}$ & $0(0)$ & $0(0)$ & $0(0)$ & $0(0)$ & $0(0)$ & $0(0)$ & & $0(0)$ & $0(0)$ & \\
\hline Viral diseases & $\begin{array}{l}142 \\
(6.4)\end{array}$ & $\begin{array}{l}92 \\
(4.1)\end{array}$ & $39(1.8)$ & $\begin{array}{l}37 \\
(1.7)\end{array}$ & $21(0.9)$ & $\begin{array}{l}8 \\
(0.4)\end{array}$ & $\begin{array}{l}9 \\
(0.4)\end{array}$ & $\begin{array}{l}25 \\
(1.1)\end{array}$ & $X^{2}=16.5$ & $0(0)$ & $0(0)$ & \\
\hline Kennel cough & $7(0.3)$ & $\begin{array}{l}4 \\
(0.2)\end{array}$ & $1(0)$ & $0(0)$ & $0(0)$ & $0(0)$ & $\begin{array}{l}2 \\
(0.1)\end{array}$ & $4(0.2)$ & $\begin{array}{l}P= \\
0.020 \text { * }\end{array}$ & $0(0)$ & $0(0)$ & / \\
\hline Parvovirosis & $\begin{array}{l}135 \\
(6.1)\end{array}$ & $88(4)$ & $38(1.7)$ & $\begin{array}{l}37 \\
(1.7)\end{array}$ & $21(0.9)$ & $\begin{array}{l}8 \\
(0.4)\end{array}$ & $\begin{array}{l}7 \\
(0.3)\end{array}$ & $\begin{array}{l}21 \\
(0.9)\end{array}$ & & $0(0)$ & $0(0)$ & \\
\hline $\begin{array}{l}\text { Convenience } \\
\text { surgery }\end{array}$ & $8(0.4)$ & $\begin{array}{l}7 \\
(0.3)\end{array}$ & $2(0.1)$ & $9(0.4)$ & $2(0.1)$ & $1(0)$ & $1(0)$ & $6(0.3)$ & $X^{2}=37.3$ & $\begin{array}{l}8 \\
(4.6)\end{array}$ & $14(8)$ & $X^{2}=3.8$ \\
\hline
\end{tabular}




\begin{tabular}{|c|c|c|c|c|c|c|c|c|c|c|c|c|}
\hline \multirow[t]{2}{*}{ Clinical diseases } & \multicolumn{9}{|l|}{ Dogs } & \multicolumn{3}{|l|}{ Cats } \\
\hline & $\begin{array}{l}\text { Cross } \\
\text { (Mixed) }\end{array}$ & $\begin{array}{l}\text { Local } \\
\text { breed }\end{array}$ & $\begin{array}{l}\text { German } \\
\text { Shepherd }\end{array}$ & $\begin{array}{l}\text { Toy } \\
\text { breeds } \\
+\end{array}$ & Rottweiler & $\begin{array}{l}\text { Cane } \\
\text { corso }\end{array}$ & $\begin{array}{l}\text { Great } \\
\text { Dane }\end{array}$ & $\begin{array}{l}\text { Other } \\
\text { exotics } \\
\neq\end{array}$ & p-value & $\begin{array}{l}\text { Exotic } \\
\text { Cat }\end{array}$ & $\begin{array}{l}\text { Local } \\
\text { Cat }\end{array}$ & $\begin{array}{l}\text { P- } \\
\text { Value }\end{array}$ \\
\hline Castration & $2(0.1)$ & $\begin{array}{l}5 \\
(0.2)\end{array}$ & $1(0)$ & $6(0.3)$ & $0(0)$ & $0(0)$ & $1(0)$ & $1(0)$ & $P=0.111$ & $\begin{array}{l}4 \\
(2.3)\end{array}$ & $\begin{array}{l}10 \\
(5.7)\end{array}$ & $\begin{array}{l}P= \\
0.143\end{array}$ \\
\hline Otectomy & $0(0)$ & $0(0)$ & $0(0)$ & $1(0)$ & $0(0)$ & $0(0)$ & $0(0)$ & $0(0)$ & & $0(0)$ & $0(0)$ & \\
\hline Ovariectomy & $0(0)$ & $1(0)$ & $0(0)$ & $0(0)$ & $0(0)$ & $0(0)$ & $0(0)$ & $1(0)$ & & $\begin{array}{l}4 \\
(2.3)\end{array}$ & $\begin{array}{l}2 \\
(1.1)\end{array}$ & \\
\hline Ovariohysterectomy & $0(0)$ & $1(0)$ & $0(0)$ & $0(0)$ & $0(0)$ & $1(0)$ & $0(0)$ & $3(0.1)$ & & $0(0)$ & $\begin{array}{l}2 \\
(1.1)\end{array}$ & \\
\hline Tail docking & $6(0.3)$ & $0(0)$ & $1(0)$ & $2(0.1)$ & $2(0.1)$ & $0(0)$ & $0(0)$ & $1(0)$ & & $0(0)$ & $0(0)$ & \\
\hline $\begin{array}{l}\text { Vaccination \& } \\
\text { health check up }\end{array}$ & $\begin{array}{l}209 \\
(9.4)\end{array}$ & $\begin{array}{l}196 \\
(8.8)\end{array}$ & $108(4.9)$ & $\begin{array}{l}69 \\
(3.1)\end{array}$ & $49(2.2)$ & $\begin{array}{l}32 \\
(1.4)\end{array}$ & $\begin{array}{l}12 \\
(0.5)\end{array}$ & $\begin{array}{l}127 \\
(5.7)\end{array}$ & I & $\begin{array}{l}64 \\
(36.6)\end{array}$ & $\begin{array}{l}25 \\
(14.3)\end{array}$ & I \\
\hline Overall prevalence & $\begin{array}{l}705 \\
(31.7)\end{array}$ & $\begin{array}{l}537 \\
(24.2)\end{array}$ & $\begin{array}{l}291 \\
(13.1)\end{array}$ & $\begin{array}{l}193 \\
(8.7)\end{array}$ & $118(5.3)$ & $\begin{array}{l}71 \\
(3.2)\end{array}$ & $\begin{array}{l}48 \\
(2.2)\end{array}$ & $\begin{array}{l}259 \\
(11.7)\end{array}$ & $\begin{array}{l}2222 \\
(100)\end{array}$ & $\begin{array}{l}79 \\
(45.1)\end{array}$ & $\begin{array}{l}96 \\
(54.9)\end{array}$ & $\begin{array}{l}175 \\
(100)\end{array}$ \\
\hline
\end{tabular}

The highest prevalence of diseases in dog were found in Cross breed (31.7\%), local breed (24.02\%), German shepherd (13.1\%), Toy breeds (8.7\%), Rottweiler (5.3\%), Cane corso (3.2\%) and Great dane (2.2\%), with a significant difference $(p<0.0001$, Table 3 and Fig. 3$)$. Cross dog breeds was significantly affected by leptospirosis, cataract, dermatitis, gastroenteritis, metritis and poisoning $(p<0.0001)$, by helminthoses and ectoparasitism $(p=$ $0.001)$, amoebiasis and babesiosis $(p=0.017)$, kennel cough and parvovirosis $(p=0.020)$. Local dog breeds were significantly affected by constipation, abscess, ascites, haematoma, paralysis, umbilical hernia and myiasis. Similarly, German shepherds were more prone to be affected by septicemia, aural haematoma, eczema, orchitis, otitis and mites (mange). Prevalence of clinical diseases in cats in relation with the breed revealed that the highest clinical conditions were found $96(54.9 \%)$ in the local cat breed in comparison with exotic cat $(79,45.1 \%)$.

Effects of age on the cases diagnosed (Table 4 and Fig. 4) 
Table 4

Age-wise prevalence of clinical diseases of dog and cat registered in private veterinary clinics

\begin{tabular}{|c|c|c|c|c|c|c|c|c|}
\hline \multirow[t]{2}{*}{ Clinical diseases } & \multicolumn{3}{|l|}{ Dogs, n (\%) } & \multirow[t]{2}{*}{ P-Value } & \multicolumn{3}{|l|}{ Cats, n (\%) } & \multirow[t]{2}{*}{ P-Value } \\
\hline & $\begin{array}{l}\text { Puppies } \\
\text { (up to } \\
1 \text { years) }\end{array}$ & $\begin{array}{l}\text { Juvenile } \\
\text { (1 to } \\
2 \text { years) }\end{array}$ & $\begin{array}{l}\text { Adult } \\
\text { (> } \\
2 \text { years) }\end{array}$ & & $\begin{array}{l}\text { Young }< \\
1 \text { year }\end{array}$ & $\begin{array}{l}\text { Adult } 1 \text { to } \\
2 \text { years }\end{array}$ & $\begin{array}{l}\text { Old > } \\
2 \text { years }\end{array}$ & \\
\hline Bacterial/fungal diseases & $18(0.8)$ & $0(0)$ & $12(0.5)$ & $X^{2}=11.1$ & $0(0)$ & $0(0)$ & $0(0)$ & \\
\hline Erlichiosis & $3(0.1)$ & $0(0)$ & $2(0.1)$ & $\begin{array}{l}P= \\
0.011 *\end{array}$ & $0(0)$ & $0(0)$ & $0(0)$ & \\
\hline Leptospirosis & $7(0.3)$ & $0(0)$ & $0(0)$ & & $0(0)$ & $0(0)$ & $0(0)$ & l \\
\hline Ringworm & $3(0.1)$ & $0(0)$ & $0(0)$ & & $0(0)$ & $0(0)$ & $0(0)$ & \\
\hline Septicemia & $5(0.2)$ & $0(0)$ & $10(0.5)$ & & $0(0)$ & $0(0)$ & $0(0)$ & \\
\hline Non specific diseases & $413(18.6)$ & $24(1.1)$ & $\begin{array}{l}121 \\
(5.4)\end{array}$ & $X^{2}=122.9$ & $29(16.6)$ & $1(0.6)$ & $4(2.3)$ & $X^{2}=30.2$ \\
\hline Abscess & $5(0.2)$ & $0(0)$ & $1(0)$ & $\begin{array}{l}P< \\
0.0001 *\end{array}$ & $1(0.6)$ & $0(0)$ & $0(0)$ & $\begin{array}{l}P= \\
0.066\end{array}$ \\
\hline Ascites & $5(0.2)$ & $0(0)$ & $0(0)$ & & $0(0)$ & $0(0)$ & $0(0)$ & \\
\hline Aural haematoma & $15(0.7)$ & $1(0)$ & $4(0.2)$ & & $2(1.1)$ & $0(0)$ & $0(0)$ & \\
\hline Bronchitis & $2(0.1)$ & $0(0)$ & $0(0)$ & & $0(0)$ & $0(0)$ & $0(0)$ & \\
\hline Cataract & $2(0.1)$ & $0(0)$ & $1(0)$ & & $0(0)$ & $0(0)$ & $0(0)$ & \\
\hline Cherry eye & $1(0)$ & $0(0)$ & $0(0)$ & & $0(0)$ & $0(0)$ & $0(0)$ & \\
\hline Conjunctivitis & $3(0.1)$ & $0(0)$ & $2(0.1)$ & & $0(0)$ & $0(0)$ & $0(0)$ & \\
\hline Constipation & $2(0.1)$ & $0(0)$ & $3(0.1)$ & & $0(0)$ & $0(0)$ & $0(0)$ & \\
\hline Dermatitis & $40(1.8)$ & $7(0.3)$ & $16(0.7)$ & & $5(2.9)$ & $0(0)$ & $0(0)$ & \\
\hline Eczema & $14(0.6)$ & $1(0)$ & $4(0.2)$ & & $2(1.1)$ & $0(0)$ & $0(0)$ & \\
\hline Edema & $3(0.1)$ & $0(0)$ & $0(0)$ & & $0(0)$ & $0(0)$ & $0(0)$ & \\
\hline Epileptiform crisis & $0(0)$ & $0(0)$ & $2(0.1)$ & & $0(0)$ & $0(0)$ & $0(0)$ & \\
\hline Epistasis & $1(0)$ & $0(0)$ & $0(0)$ & & $1(0.6)$ & $0(0)$ & $0(0)$ & \\
\hline Gastroenteritis & $156(7)$ & $7(0.3)$ & $11(0.5)$ & & $2(1.1)$ & $0(0)$ & $0(0)$ & \\
\hline Haemorrhage & $0(0)$ & $0(0)$ & $1(0)$ & & $0(0)$ & $0(0)$ & $0(0)$ & \\
\hline Hematoma & $2(0.1)$ & $0(0)$ & $5(0.2)$ & & $0(0)$ & $0(0)$ & $0(0)$ & \\
\hline Mastitis & $2(0.1)$ & $0(0)$ & $1(0)$ & & $0(0)$ & $0(0)$ & $0(0)$ & \\
\hline Metritis/pyometra & $3(0.1)$ & $0(0)$ & $0(0)$ & & $0(0)$ & $0(0)$ & $0(0)$ & \\
\hline Orchitis & $6(0.3)$ & $2(0.1)$ & $2(0.1)$ & & $0(0)$ & $0(0)$ & $0(0)$ & \\
\hline Otitis & $14(0.6)$ & $2(0.1)$ & $9(0.4)$ & & $0(0)$ & $1(0.6)$ & $1(0.6)$ & \\
\hline Paralysis & $1(0)$ & $0(0)$ & $3(0.1)$ & & $1(0.6)$ & $0(0)$ & $1(0.6)$ & \\
\hline Paraphimosis & $2(0.1)$ & $0(0)$ & $0(0)$ & & $0(0)$ & $0(0)$ & $0(0)$ & \\
\hline Pneumonia & $5(0.2)$ & $0(0)$ & $0(0)$ & & $0(0)$ & $0(0)$ & $0(0)$ & \\
\hline Poisoning & $121(5.4)$ & $2(0.1)$ & $51(2.3)$ & & $9(5.1)$ & $0(0)$ & $0(0)$ & \\
\hline Rectal prolapse & $0(0)$ & $0(0)$ & $0(0)$ & & $2(1,1)$ & $0(0)$ & $0(0)$ & \\
\hline Tumor & $2(0.1)$ & $2(0.1)$ & $5(0.2)$ & & $2(1.1)$ & $0(0)$ & $2(1.1)$ & \\
\hline Umbilical hernia & $3(0.1)$ & $0(0)$ & $0(0)$ & & $0(0)$ & $0(0)$ & $0(0)$ & \\
\hline Urinary infection & $3(0.1)$ & $0(0)$ & $0(0)$ & & $2(1.1)$ & $0(0)$ & $0(0)$ & \\
\hline Non-infectious diseases & $61(2.7)$ & $5(0.2)$ & $15(0.7)$ & $X^{2}=15.8$ & $13(7.4)$ & $0(0)$ & $0(0)$ & \\
\hline
\end{tabular}




\begin{tabular}{|c|c|c|c|c|c|c|c|c|}
\hline \multirow[t]{2}{*}{ Clinical diseases } & \multicolumn{3}{|l|}{ Dogs, $n(\%)$} & \multirow[t]{2}{*}{ P-Value } & \multicolumn{3}{|l|}{ Cats, n (\%) } & \multirow[t]{2}{*}{ P-Value } \\
\hline & $\begin{array}{l}\text { Puppies } \\
\text { (up to } \\
1 \text { years) }\end{array}$ & $\begin{array}{l}\text { Juvenile } \\
\text { (1 to } \\
2 \text { years) }\end{array}$ & $\begin{array}{l}\text { Adult } \\
\text { (> } \\
2 \text { years) }\end{array}$ & & $\begin{array}{l}\text { Young }< \\
1 \text { year }\end{array}$ & $\begin{array}{l}\text { Adult } 1 \text { to } \\
2 \text { years }\end{array}$ & $\begin{array}{l}\text { Old }> \\
2 \text { years }\end{array}$ & \\
\hline Accidental injury & $15(0.7)$ & $1(0)$ & $8(0.4)$ & $P=0.104$ & $5(2.9)$ & $0(0)$ & $0(0)$ & \\
\hline Dislocation & $3(0.1)$ & $0(0)$ & $0(0)$ & & $0(0)$ & $0(0)$ & $0(0)$ & / \\
\hline food hypersensitivity & $11(0.5)$ & $1(0)$ & $0(0)$ & & $1(0.6)$ & $0(0)$ & $0(0)$ & \\
\hline Fracture & $11(0.5)$ & $2(0.1)$ & $1(0)$ & & $4(2.3)$ & $0(0)$ & $0(0)$ & \\
\hline Sprain & $2(0,1)$ & $1(0)$ & $0(0)$ & & $0(0)$ & $0(0)$ & $0(0)$ & \\
\hline Wounds & $19(0.9)$ & $0(0)$ & $6(0.3)$ & & $3(1.7)$ & $0(0)$ & $0(0)$ & \\
\hline Parasitic diseases & $216(9.7)$ & $22(1)$ & $45(2)$ & $X^{2}=2.7$ & $15(8.6)$ & $2(1.1)$ & $0(0)$ & \\
\hline Helminthoses & $140(6.3)$ & $14(0.6)$ & $29(1.3)$ & $P=0.836$ & $15(8.6)$ & $2(1.1)$ & $0(0)$ & I \\
\hline Mites (Mange) & $12(0.5)$ & $2(0.1)$ & $1(0)$ & & $0(0)$ & $0(0)$ & $0(0)$ & \\
\hline Myiasis & $25(1.1)$ & $1(0)$ & $6(0.3)$ & & $0(0)$ & $0(0)$ & $0(0)$ & \\
\hline Tick/Flea infestation & $39(1.8)$ & $5(0.2)$ & $9(0.4)$ & & $0(0)$ & $0(0)$ & $0(0)$ & \\
\hline Protozoan diseases & $34(1.5)$ & $8(0.4)$ & $17(0.8)$ & $X^{2}=1.1$ & $0(0)$ & $0(0)$ & $0(0)$ & \\
\hline Amoebiasis & $21(0.9)$ & $4(0.2)$ & $8(0.4)$ & $P=0.569$ & $0(0)$ & $0(0)$ & $0(0)$ & I \\
\hline Babesiosis & $13(0.6)$ & $4(0.2)$ & $9(0.4)$ & & $0(0)$ & $0(0)$ & $0(0)$ & \\
\hline Viral diseases & $329(14.8)$ & $15(0.7)$ & $29(1.3)$ & $X^{2}=60.4$ & $0(0)$ & $0(0)$ & $0(0)$ & \\
\hline Kennel cough & $8(0.4)$ & $0(0)$ & $10(0.5)$ & $\begin{array}{l}\mathrm{P}< \\
0.0001^{*}\end{array}$ & $0(0)$ & $0(0)$ & $0(0)$ & I \\
\hline Parvovirosis & $321(14.4)$ & $15(0.7)$ & $19(0.9)$ & & $0(0)$ & $0(0)$ & $0(0)$ & \\
\hline Convenience surgery & $28(1.3)$ & $2(0.1)$ & $6(0.3)$ & $X^{2}=5.2$ & $20(11.4)$ & $2(1.1)$ & $0(0)$ & $X^{2}=0.7$ \\
\hline Castration & $12(0.5)$ & $0(0)$ & $4(0.2)$ & $P=0.730$ & $13(7.4)$ & $1(0.6)$ & $0(0)$ & $\begin{array}{l}P= \\
0.711\end{array}$ \\
\hline Otectomy & $1(0)$ & $0(0)$ & $0(0)$ & & $0(0)$ & $0(0)$ & $0(0)$ & \\
\hline Ovariectomy & $2(0.1)$ & $0(0)$ & $0(0)$ & & $5(2.9)$ & $1(0.6)$ & $0(0)$ & \\
\hline Ovariohysterectomy & $3(0.1)$ & $1(0)$ & $1(0)$ & & $2(1.1)$ & $0(0)$ & $0(0)$ & \\
\hline Tail docking & $10(0.5)$ & $1(0)$ & $1(0)$ & & $0(0)$ & $0(0)$ & $0(0)$ & \\
\hline $\begin{array}{l}\text { Vaccination and health } \\
\text { check up }\end{array}$ & $428(19.3)$ & $151(6.8)$ & $223(10)$ & l & $55(31.4)$ & $19(10.9)$ & $15(8.6)$ & I \\
\hline Overall prevalence & $1527(68.7)$ & $227(10.2)$ & $\begin{array}{l}468 \\
(21.1)\end{array}$ & $\begin{array}{l}2222 \\
(100)\end{array}$ & $132(75.4)$ & $24(13.7)$ & 19 (10.9) & $\begin{array}{l}175 \\
(100)\end{array}$ \\
\hline
\end{tabular}

Dogs were divided into age groups as puppies, juvenile and adult whereas cats were divided as young, adult and old. The age wise highest prevalence of diseases in dog was observed in puppies (68.7\%), followed by adult $(21.1 \%)$ and juvenile $(10.2 \%)$ with a significant difference $(p<0.0001$, Table 4 and Fig. 4). Parvovirosis (14.4\%), poisoning (5.4\%), gastroenteritis $(7 \%)$ and dermatitis $(1.8 \%)$ were found affecting significantly $(P<0.05)$ the puppies compared to the other age groups. Unlike in the puppies, adult dogs were significantly $(P<0.05)$ affected by septicemia $(0.5 \%)$, hematoma $(0.2 \%)$, tumor $(0.2 \%)$ and kennel cough $(0.5 \%)$. Prevalence of clinical condition was high in young cats $(75.5 \%)$ and adult cats $(13.7 \%)$, but lowest in old cats (10.9\%). No significant association between age group and diagnosed disease cases was observed in cats.

\section{Discussion}

To the best of our knowledge, the present study had given an overall idea about the prevalence of the most common occurred disease conditions and associated clinical signs amongst dogs and cats, which were registered in private veterinary clinics in Doula city, Cameroon, during 5 years period from 2014 to 2019. It is the first systematic assessment on quantitative estimates of clinical diseases and conditions in pet dogs and cats in Cameroon. Among the 2397 clinical cases diagnosed in this study, $92.7 \%$ were from dogs and $7.3 \%$ from cats, which disagree the results found elsewhere characterized by a more presence of cat disease cases $[3,18-20]$. This can be explained as there was a magnificent difference in geographical 
distribution and the period of the studies. In addition, there is a tendency of population to own dogs as guard because of the level of insecurity in Douala city. Dog disease cases were found largely more represented than surgical cases, vaccinations and health checkups, which is consistent with earlier reports $[3,18]$. Digestive disorders (anorexia, diarrhea, vomiting, abdominal bloating, hypersalivation and abdominal pain) and skin disorders (pruritis and ectoparasites) were found to be the highest clinical disorders in both dogs and cats with the frequency of $58 \%$ and $13.5 \%$, respectively. Similar findings were reported in a study in Egypt, and the recorded prevalence in dogs were $56.5 \%$ and $27.86 \%$ for digestive problems and dermatological problems, respectively [21]. Dermatological disorders accounted for $18.8 \%$ and $15.2 \%$, respectively of all the dogs and cats examined in University of Montreal, Quebec [22]. In contrast, results found in Mekelle City, Ethiopia, showed that 38.5\% of the dogs were affected by skin problem and gastrointestinal related cases were the second major health problems with a frequency of 33.0\% [15]. The high frequency of anorexia (21.5\%) in this study can be explained as anorexia is actually the most common presented complaint for a myriad of disease processes with wide variety of etiology and pathogenesis [23].

Amongst the 2397 clinical records examined in various private veterinary clinics, a total of 46 types of clinical diseases were diagnosed in both dogs and cats. Infectious diseases were found to be a major cause of illness in dogs with a high prevalence of $33.6 \%$, which were also the case in the reports on dog diseases in some States in Nigeria, and young dog diseases in Grand Tunis, Tunisia [24-26]. Overall prevalence shows that parvovirosis occupied highest prevalence in dogs (16\%), followed by helminthoses (8.2\%), gastroenteritis (7.8\%), poisoning (7.8\%), dermatitis (2.8\%) and babesiosis (1.2\%), which corroborate the results find elsewhere [24, 26, 27]. Parvoviral exposure appeared to be ubiquitous within the dog and cat populations [28]. Several prevalence were reported for canine parvovirosis as $22.7 \%$ [29], 32.14\% [30], 21.3\% [27], 5.7\% [31] and 13.4\% [32], with vomiting, anorexia, bloody foul smelling diarrhea, dehydration, mucosal pallor, sunken eyes, abdominal pain, depression and weakness being the main constantly clinical signs [33]. Furthermore, canine parvovirus enteritis 47(19.5\%) and gastroenteritis 31(12.9\%) have been recorded as the most frequent causes of mortality in dogs in Delta State, Nigeria [34]. Concerning dog helminthoses, prevalence were found $85.5 \%$ in other area of Cameroon [14], 86.54\% in central Ethiopia [35], 80\% in Lahore Area [27], 49.95\% in North-Eastern Nigeria [36], 19.19\% in Maiduguri, Nigeria [37], 12.9\% in Paris region [38] and 16.5\% in Calgary, Alberta [39], With Ancylostoma caninum, Dipylidium caninum, Toxocara canis, Strogyloides stercoralis, Taenia spp, Echinococcus granulosus and Isospora canis being the most reported species in tropical region. The difference in the prevalence between our study and these authors could be attributed to geographical areas, the different diagnostic techniques employed, health cares and animal management practices followed. Considering the public health implication of these helminths, preventive measures should be taken to reduce the risk of transmission to humans. These include routine and adequate deworming exercise, proper care of hygienic environment of pets, patronizing of qualified veterinary doctors and establishment of programs centered on enlightening the pet owners of possible zoonotic parasitic diseases [40]. Prevalent clinical diseases in cats included helminthoses (9.7\%), poisoning (5.1\%), dermatitis (2.9\%), accidental injury (2.9\%) and fracture (2.3\%). Similarly, high prevalence of helminthoses (26.67\%) was also found in cats in Maiduguri state, Nigeria, by William et al. [37], whereas smaller prevalence were reported elsewhere [3, 18, 38].

In the present study a significant effect $(p=0.009)$ of sex on the occurrence of the clinical disease conditions was found and this finding was in agreement with another studies, as male dogs were rendered at high risk for developing common canine digestive problems [21], canine toxicosis [41], ectoparasitic and endoparasitic infections [42], infectious diseases [26], common young dog diseases [25] and some other disease conditions [3, 6, 24]. However, some studies have reported a high prevalence of female diseases as compared to male [36, 43-45], while other was suggesting no effect of the sex on specific canine illnesses [46]. Most of the pet owners reared male pets rather than females due to pet owners unaware about the reproductive physiology of female dogs and cats. Dog owners do provides better veterinary care for males than the females, probably due to their use as guard dogs. Male dogs were found to be more affected with aural haematoma, dermatitis, eczema, gastroenteritis, orchitis, poisoning and wounds. Similar finding was reported for aural haematoma, orchitis and poisoning [24]. Orchitis is a clinical condition of male dogs. The predisposing factors in male dogs poisoning may be related to their use as guard dogs and presenting protector behaviors, which risk them as targets to criminal poisoning.

In the present work, breed had a significant effect $(p<0.0001)$ on the distribution of clinical diseases in pets. Of the dog breeds, Cross breed $(31.7 \%)$, local breed (24.02\%), German shepherd (13.1\%), Toy breeds (8.7\%), Rottweiler (5.3\%), Cane corso (3.2\%) and Great Dane (2.2\%) were mostly affected. This result agrees with other findings reported elsewhere $[6,26,31,43,47]$. Mixed dog breeds were significant $(p<0.05)$ for gastroenteritis, poisoning, helminthoses, ectoparasitism, kennel cough and parvovirosis. This corroborates the finding of Shima et al. [41], who also reported a higher incidence of poisoning in mixed breeds (29\%), Alsatian (25\%), Rottweiler (16\%) compare with the others. Nonetheless, Shima et al. [24] were reporting a significantly high prevalence of helminthoses, septicemia and ectoparasites in Alsatian. In general, the occurrence of ectoparasitism, parvovirosis, and helminthoses might relate to management issues such as keeping dogs on free range, insufficient housing, inadequate deworming practices, inadequate vaccination program and inadequate ectoparasites control practices. Prevalence of clinical diseases in cats revealed that local cats were more prone to diseases than were with exotic cats and this result agree with Parvez et al. [3]. However Sarker et al. [18], found the same prevalence in both local and exotic cat breeds.

In agreement with the findings of other workers, the age of pets influenced their chances of developing particular disease condition. Age-wise prevalence of the dog presented with various clinical conditions shows that $68.7 \%$ were puppies, followed by adult (21.1\%) and juvenile (10.2\%), which is in accordance with several studies [6, 24, 26, 29,41]. puppies and adult dogs apparently received better veterinary care or had a higher incidence of diseases compared with the juvenile probably due to their age and immune status. For instance, parvovirosis (14.4\%), poisoning (5.4\%), gastroenteritis (7\%) and dermatitis (1.8\%) were found affecting significantly $(P<0.05)$, the puppies compared to the other age groups. Similar results were reported for parvovirosis and gastroenteritis [15, 24, 29-32, 46, 48], and poisoning [41]. The most likely reason that dogs younger than six months are at the greatest risk of parvovirosis is very high affinity of canine parvovirus for tissues with high mitotic activity. This may also be as a result of the fact that puppies don't have a well-developed immune status (maternally derived antibodies) which eventually begins to wane down at about 12 weeks of age thus 
opening a window for many clinical conditions to occur in them [49]. On the other hand, higher incidence on poisoning in younger dogs could be possible because they tend to be more active, and could be easily poisoned by lower doses of toxicants due to immature neutralizing enzyme systems [41]. Prevalence of clinical condition were high in young cats (75.5\%) and adult cats (13.7\%), but lowest in old cats (10.9\%), which agree with finding reported by Sarker et al. [18].

\section{Conclusion}

The objective of the current survey was to deliver fundamental information on the prevalence of clinical diseases and other clinical conditions of dogs and cats in Doula city, Cameroon. Most of the clinical diseases presented to the veterinary clinics were avoidable cases. It was concluded that digestive problems considered as the major health problem among owned dogs and cats. Sex, breed and age had a significant effect on the distribution of pet clinical diseases. This study revealed that males were more affected than females. Cross dog breeds were mostly affected, while highest clinical diseases were found in the local cat breed. Puppies and young cats were more prone to diseases. It is suggested that the pets should be given appropriate attention for their vaccination, deworming, ectoparasites control measures and other health precautions as an attempts to prevent and eradicate zoonotic diseases. Edification of dog owners on preventive measures will help in alleviating some of these health issues. Government have his role to play by formulating policies that will encourage pet owners to vaccinate and adequately care for their pets as well as patronized competent veterinarian. The present data allow the veterinarians in Cameroon to ascertain their needs for diagnostic utensils and medication that are require at any pet clinic.

\section{Material And Methods}

\section{Study Design:}

A retrospective study over a period of 5 years was carried out to assess the major health problems of dogs and cats, and to determine the risk factors associated with the disease conditions of pets that are brought to the clinic.

\section{Study area:}

The study was conducted from 2014 to 2019 in Doula city, the largest city in Cameroon and its economic capital. As from 2018 , the city and its surrounding area had an estimated population of 2,768,400. Douala typically features warm and humid conditions with an average annual temperature of $27.0^{\circ} \mathrm{C}$ and an average humidity of $83 \%$. Douala sees plentiful rainfall during the course of the year, experiencing on average roughly 3,600 millimeters of precipitation per year. The city sits on the estuary of Wouri River at $009^{\circ} 41^{\prime} \mathrm{E}$ and $04^{\circ} 03^{\prime} \mathrm{N}$ latitude, and an altitude of $13 \mathrm{~m}$ above sea level. The area has substantial population of dogs, which are used as pets, for security and means of livelihood.

\section{Clinical evaluation/Data collection}

Data of diagnosed clinical cases/conditions of dogs and cats presented at the various veterinary clinics were collected in Douala city. The selection criteria of each clinic were based on consistent records kept for the studied period. Diagnosis was based on complaint of the pet owners, complete medical history, physical examination, clinical symptoms, and sometimes post-mortem findings. Detailed clinical examination was performed using visual examination, rectal temperature, pulse, respiration rates, and examination of different organs and systems using palpation, percussion, and auscultation when needed [50]. When necessary, some disease cases were confirmed in the laboratory by blood and fecal examination. The diagnosis made for each animal was classified into one of the following categories; bacterial/fungal diseases, non-infectious diseases, parasitic diseases, nonspecific diseases (multicausal diseases), protozoan diseases and viral diseases. Data collected for each dog and cat examined during data collection periods included clinic-specific patient identifier, species, birth date, breed, sex and assigned diagnostic.

\section{Data Analysis:}

All the data obtained from clinics were loaded, organized, structure and stored on the Microsoft excel spreadsheet in which descriptive statistics (in percentages) were conducted. Statistical tests were conducted using statistical package for social sciences (SPSS) version 26. Chi-square test was used to examine whether the pets' sex, age and breed were associated with any of the disease cases. For all statistical analysis, a significant level ( $p$ value) of less than 0.05 was considered as statistically significant.

\section{Declarations}

- Ethics approval and consent to participate: Not applicable.

- Consent for publication: Not applicable.

- Availability of data and materials: The datasets generated and/or used during the current study can be requested via email from the corresponding author; Dr. Kana Dongmo Alain Gildas (kanadongmo1@gmail.com)

- Competing interests: The authors declare that there is no existent competing of interest.

- Funding: No funding was received for the completion of the current study. 
- Authors' Contributions: It is clearly stated that all authors has contributed significantly to the study. AGDK and CCZD analyzed and interpreted the results. AGDK drafted the manuscript with contribution from all authors. JK supervised running of the project. All authors read and approved the final manuscript.

- Acknowledgements: The authors thank all the veterinarians and staff of the various veterinary clinics in Doula city for giving access to the relevant clinical records used for this study. Without their help, participation and cooperation, this study would not have been possible.

\section{References}

1. Podberscek AL. Positive and Negative Aspects of Our Relationship with Companion Animals. Vet Res Commun. 2006;30(Suppl. 1):21-7. https://doi.org/10.1007/s11259-006-0005-0.

2. Robertson ID, Irwin PJ, Lymbery AJ, Thompson RCA. The role of companion animals in the emergence of parasitic zoonoses. Int J Parasitol. 2000;30(12):1369-77. https://doi.org/10.1016/S0020-7519(00)00134-X.

3. Parvez MA, Prodhan MAM, Das BC, Khatun R. Prevalence of Clinical Conditions in Dogs and Cats at Teaching Veterinary Hospital (TVH) in Chittagong Veterinary and Animal Sciences University, Bangladesh. Research Journal for Veterinary Practitioners. 2014;2(6):99-104. https://doi.org/10.14737/journal.rjvp/2014/2.6.99.104.

4. Headey B, Grabka M: Pet ownership is good for your health and saves public expenditure too. In: The Australian Social Monitor. Volume 5, edn.: Melbourne Institute of Applied Economic and Social Research; 2000.

5. McNicholas J, Gilbey A, Rennie A, Ahmedzai S, Dono J-A, Ormerod E. Pet ownership and human health: a brief review of evidence and issues. BMJ. 2005;331(7527):1252-4. https://doi.org/10.1136/bmj.331.7527.1252.

6. Islam O, Khatun S, Famous M. Comparative Hospital Prevalence Study of Different Diseases of Dog at Dhaka, Chittagong and Sylhet District of Bangladesh. Veterinary Sciences: Research Reviews. 2019;5(2):53-7. https://doi.org/10.17582/journal.vsrr/2019/5.2.53.57.

7. Bouli FPNO, Awah-Ndukum J, Kilekoung J-PM, Tejiokem MC, Tchoumboue J. Canine rabies epidemiology in Garoua, Ngaoundéré and Yaoundé cities, Cameroon. Pan African Medical Journal. 2018;10(10):3.

8. Njong N, Tebug S, Abia W, Yepka J. Dog-associated husbandry practices favouring the spread of zoonotic pathogens with reference to helminth parasites in Yaounde, Cameroon. Scientific Journal of Veterinary Advances. 2012;1(5):1-7.

9. Bouli FPNO, Awah-Ndukum J, Mingoas KJ-P, Tejiokem MC, Tchoumboue J. Dog demographics and husbandry practices related with rabies in Cameroon. Trop Anim Health Prod. 2019;52,:979-87. https://doi.org/10.1007/s11250-019-02085-9.

10. Awah-Ndukum J. Ecological aspects of dogs in relation to rabies control and public health significance in North-west Cameroon. Journal of the Cameroon Academy of Sciences. 2003;3(1):25-40.

11. Awah-Ndukum J, Tchoumboue J, Zoli P. Involvement of communities in the control of dog-related public health hazards in the Western Highlands of Cameroon. Journal of the Cameroon Academy of Sciences. 2004;4(1):11-8.

12. Pfukenyi DM, Chipunga SL, Dinginya L, Matenga E. A survey of pet ownership, awareness and public knowledge of pet zoonoses with particular reference to roundworms and hookworms in Harare, Zimbabwe. Tropical animal health and production 2010, 42(2):247-252. https://doi.org/10.1007/s11250-009-9413-9.

13. Woolhouse MEJ, Dye C, Cleaveland S, Laurenson MK, Taylor LH. Diseases of humans and their domestic mammals: pathogen characteristics, host range and the risk of emergence. Philosophical Transactions of the Royal Society of London Series B: Biological Sciences. 2001;356(1411):991-9. https://doi.org/10.1098/rstb.2001.0889.

14. Komtangi MC, Mpoame M, Payne V, Ngufor M. Prevalence of gastrointestinal helminths of dogs in Dschang, Cameroon. Journal of the Cameroon Academy of Sciences. 2005;5(1):11-4.

15. Gebretsadik A, Hussien D, Cruz RC. Assessment of major health problems of dogs in Mekelle City. Ethiopia Global Veterinaria. 2014;12(2):176-80. https://doi.org/10.5829/idosi.gv.2014.12.02.81145.

16. Kouamo J, Kana AGD, Zoli AP. Pratique des opérations chirurgicales et lutte contre l'infection du site opératoire chez les chiens et chats par les vétérinaires privés du Cameroun. Revue Vétérinaire Clinique. 2018;53(3):69-77. https://doi.org/10.1016/j.anicom.2018.07.003.

17. Ngah OBFP, Awah-Ndukum J, Tejiokem CM, Mingoas KJ-P. Knowledge, attitudes and practices (KAP) evaluation regarding rabies in the Centre, Adamawa and North Regions of Cameroon. Journal of Animal Science Veterinary Medicine. 2018;3(6):176-83. https://doi.org/10.31248/JASVM2018.114.

18. Sarker MS, Ahaduzzaman M, Kabir MN, Rahman MK, Hossain F, Nath SK, Bupasha ZB. Prevalence of clinical conditions in dogs and cats at central veterinary hospital (CVH) in Dhaka, Bangladesh. Van Veterinary Journal. 2015;26(2):101-5.

19. Lund EM, Armstrong PJ, Kirk CA, Kolar LM, Klausnor J. Health status and population characteristics of dogs and cats examined at private veterinary practices in the United States. Journal-American Veterinary Medical Association. 1999;214(9):1336-41.

20. Radford AD, Noble PJ, Coyne KP, Gaskell RM, Jones PH, Bryan JGE, Setzkorn C, Tierney Á, Dawson S. Antibacterial prescribing patterns in small animal veterinary practice identified via SAVSNET: the small animal veterinary surveillance network. Vet Rec. 2011;169(12):310-0.

https://doi.org/10.1136/vr.d5062. 
21. Rakha GMH, Abdl-Haleem MM, Farghali HAM, Abdel-Saeed H. Prevalence of common canine digestive problems compared with other health problems in teaching veterinary hospital, Faculty of Veterinary Medicine, Cairo University, Egypt. Veterinary world 2015, 8(3):403-411. https://doi.org/10.14202/vetworld.2015.403-411.

22. Scott DW, Paradis M. A survey of canine and feline skin disorders seen in a university practice: Small Animal Clinic, University of Montréal, SaintHyacinthe. The Canadian veterinary journal. 1990;31(12):Québec (1987-8. 830-835.

23. Delaney SJ. Management of Anorexia in Dogs and Cats. Veterinary Clinics: Small Animal Practice 2006, 36(6):1243-1249. https://doi.org/10.1016/j.cvsm.2006.08.001.

24. Shima K, Tion M, Mosugu J, Apaa T. Retrospective study of disease incidence and other clinical conditions diagnosed in owned dogs in Delta State, Nigeria. Journal of Advanced Veterinary Animal Research. 2015;2(4):435-49. https://doi.org/10.5455/javar.2015.b.115.

25. Tagorti G. Disease prevalence among young dogs in Grand Tunis, Tunisia: A retrospective study. Veterinary world. 2019;12(4):489.

26. Tion M, Zon G, Fotina H, Ogbu K, Nguetyo S, Amine A, Oochi J, Ojeamiren M, Saganuwan A. Epizootiology of Infectious Diseases of Dog in Some States in Nigeria (2015-2018). Scientific Messenger of LNU of Veterinary Medicine and Biotechnologies Series. Veterinary Sciences.

2019;21(96):33-40. https://doi.org/10.32718/nvlvet9606.

27. Jafri SA, Rabbani M. Prevalence of canine diseases in Lahore area. Pakistan Vet J. 1999;19(1):40-2.

28. Levy JK, Crawford PC, Lappin MR, Dubovi EJ, Levy MG, Alleman R, Tucker SJ, Clifford EL. Infectious Diseases of Dogs and Cats on Isabela Island, Galapagos. J Vet Intern Med. 2008;22(1):60-5. https://doi.org/10.1111/j.1939-1676.2007.0034.x.

29. Umar S, Ali A, Younus M, Maan MK, Shahzad A, Khan WA, Irfan M. Prevalence of canine parvovirus infection at different pet clinics in Lahore, Pakistan. Pakistan Journal of Zoology. 2015;47(3):657-63.

30. Tagorti G. Prevalence of canine parvovirus infection in Grand Tunis, Tunisia. Journal of Advanced Veterinary Animal Research. 2018;5(1):93-7.

31. Terzungwe TM, Thaddaeus AT, Saganuwan SA, Aondonenge TAF, Washima A-T, Samuel N. The epidemiology of canine parvovirus enteritis in dogs of Makurdi, Benue State, Nigeria. World's Veterinary Journal. 2018;8(3):48-54.

32. Shima K, Apaa T, Mosugu J. Epidemiology of Canine Parvovirus Enteritis among Hospitalized Dogs in Effurun/Warri Metropolitan Region of Delta State, Nigeria. Open Access Library Journal. 2015;02(1):1-7. https://doi.org/10.4236/oalib.1101208.

33. Mylonakis ME, Kalli I, Rallis TS. Canine parvoviral enteritis: an update on the clinical diagnosis, treatment, and prevention. Vet Med (Auckl). 2016;7:91-100. https://doi.org/10.2147/VMRR.S80971.

34. Shima FK, Mosugu JIT, Apaa TT. Causes of mortality in dogs in and around Effurun/Warri Municipality of Delta State, Nigeria. Bulletin of Animal Health Production in Africa. 2014;62(4):387-96.

35. Zewdu E, Semahegn Y, Mekibib B. Prevalence of helminth parasites of dogs and owners awareness about zoonotic parasites in Ambo town, central Ethiopia. Ethiopian Veterinary Journal. 2010;14(2):17-30.

36. Karshima S, Tizhe E, Bukar D. A retrospective study of parasitic diseases of dogs and cats in Jalingo, North-Eastern Nigeria between 1998 and 2008. Sokoto Journal of Veterinary Sciences. 2010;8(1):46-9.

37. William A, Chaudhari S, Atsandac N. Prevalence of some diseases of dogs and cats at the state government veterinary clinic in Maiduguri (Nigeria). Pakistan Veterinary Journal. 2002;22(2):56-8.

38. Beugnet F, Guillot J, Polack B, Chermette R. Enquête sur le parasitisme digestif des chiens et des chats de particuliers de la région parisienne. Revue Méd Vét. 2000;151(5):443-6.

39. Joffe D, Van Niekerk D, Gagné F, Gilleard J, Kutz S, Lobingier R. The prevalence of intestinal parasites in dogs and cats in Calgary, Alberta. The Canadian Veterinary Journal. 2011;52(12):1323-8.

40. Ola-Fadunsin SD. Parasitic diseases of dogs in Osun State, south-west Nigeria, and its zoonotic implications. Nigerian Journal of Parasitology. 2018;39(2):211-9. https://doi.org/10.4314/njpar.v39i2.17.

41. Shima KF, Gberindyer AF, Apaa TT, Mosugu IJ. Clinico-epidemiological study on canine toxicosis in Effurun/Warri Municipality region of Delta State, Nigeria. Journal of Advanced Veterinary Animal Research. 2015;2(3):357-61.

42. Mbaya A, Aliyu M, Nwosu C, Ibrahim U, Shallanguwa J. A ten-year retrospective study of the prevalence of parasitic infections of dogs at the University of Maiduguri Veterinary Teaching Hospital, Nigeria. Nigerian Veterinary Journal. 2008;29(2):31-6.

43. Rahman MM. A Retrospective Study on Dog Diseases in Sylhet City of Bangladesh. International Journal of Veterinary Science. 2017;6(3):127-30.

44. Runa N, Uddin A, Husna A, Runa N, Sabina Y, Badruzzaman ATM, Md Sadikul I, Noor M, Alam K, Rahman M. Prevalence of diseases in pet animals at Dhaka city of Bangladesh. Annals of Veterinary Animal Science. 2016;3:88-92.

45. Mahmud M, Belal S, Uddin F. Prevalence of protozoan diseases in pet dogs at district veterinary hospital, Sirajganj, Bangladesh. Bangladesh Journal of Veterinary Medicine. 2014;12(2):191-6.

46. Wells DL, Hepper PG. Prevalence of disease in dogs purchased from an animal rescue shelter. Vet Rec. 1999;144(2):35-8. https://doi.org/10.1136/vr.144.2.35.

47. Tarafder M, Samad M. Prevalence of clinical diseases of pet dogs and risk perception of zoonotic infection by dog owners in Bangladesh. Bangladesh Journal of Veterinary Medicine. 2010;8(2):163-74.

48. Gombač M, Švara T, Tadić M, Pogačnik M. Retrospective study of canine parvovirosis in Slovenia. Slovenia Veterinary Research. 2008;45(2):73-8.

Page 16/18 
49. Mila H, Grellet A, Desario C, Feugier A, Decaro N, Buonavoglia C, Chastant-Maillard S. Protection against canine parvovirus type 2 infection in puppies by colostrum-derived antibodies. Journal of Nutritional Science. 2014;3(e54):1-4. https://doi.org/10.1017/jns.2014.57.

50. Jones D: Chap. 1 - History and Physical Examination A2 - Birchard, Stephen J. In: Saunders Manual of Small Animal Practice (Third Edition). edn. Edited by Sherding RG. Saint Louis: W.B. Saunders; 2006: 1-17.

\section{Figures}

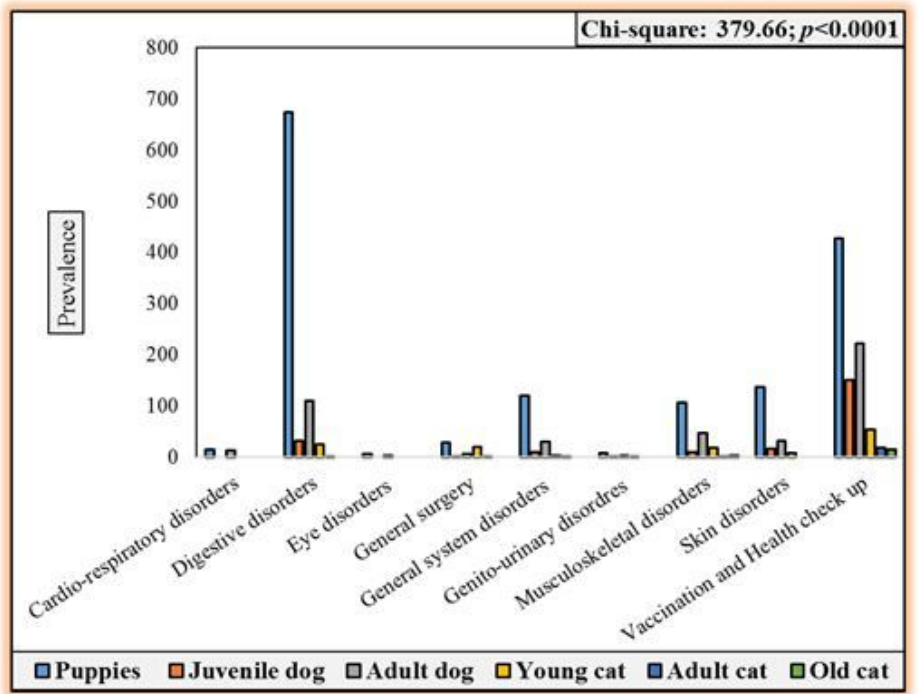

\section{Figure 1}

Prevalence of common clinical conditions in dogs and cats according to age group

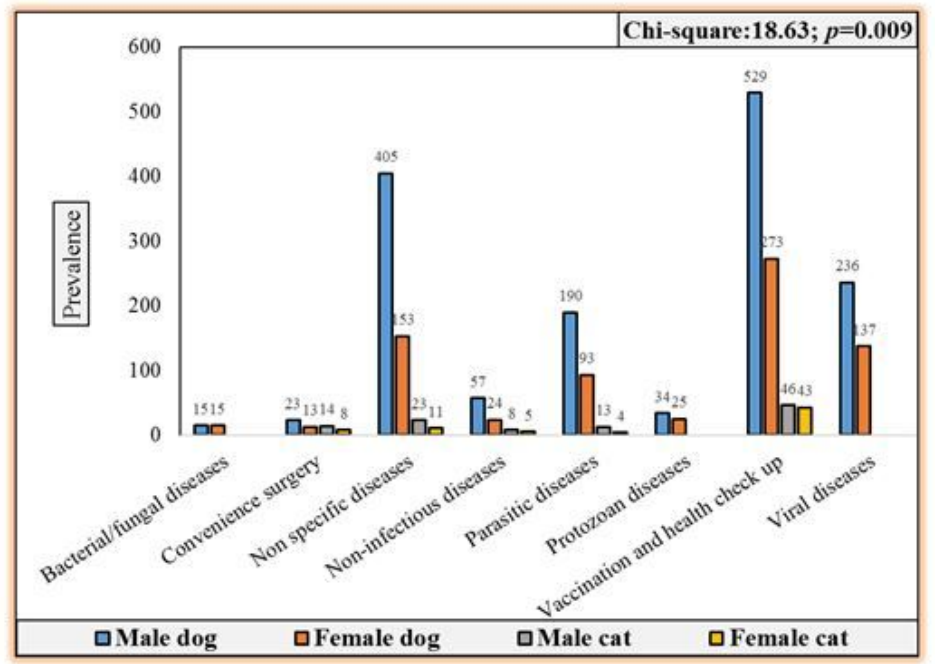

Figure 2

Sex distribution of common clinical diseases reported for 175 cats and 2222 dogs 


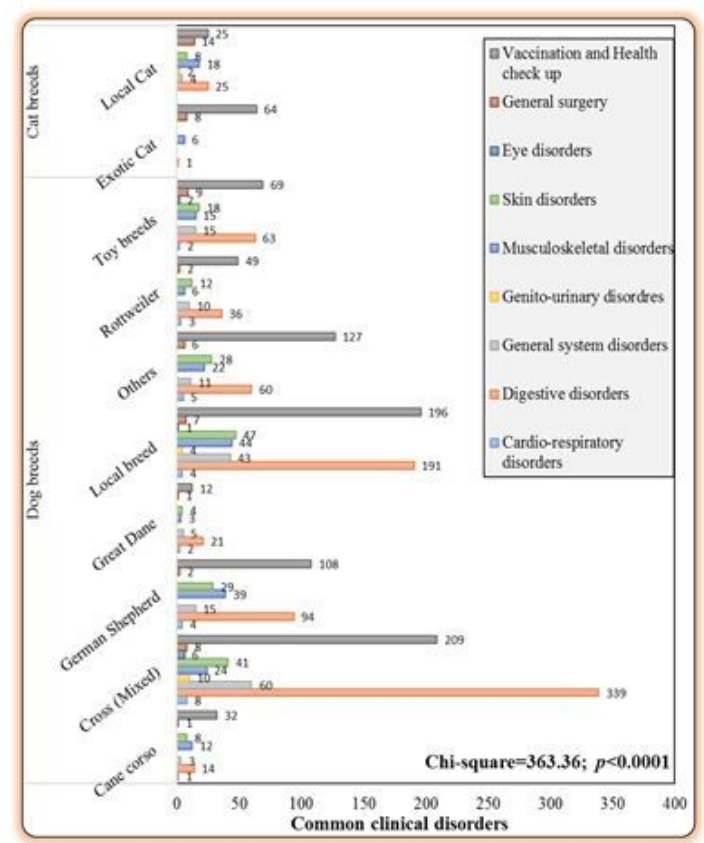

\section{Figure 3}

Breed distribution for 175 cats and 2222 dogs examined at private veterinary practices

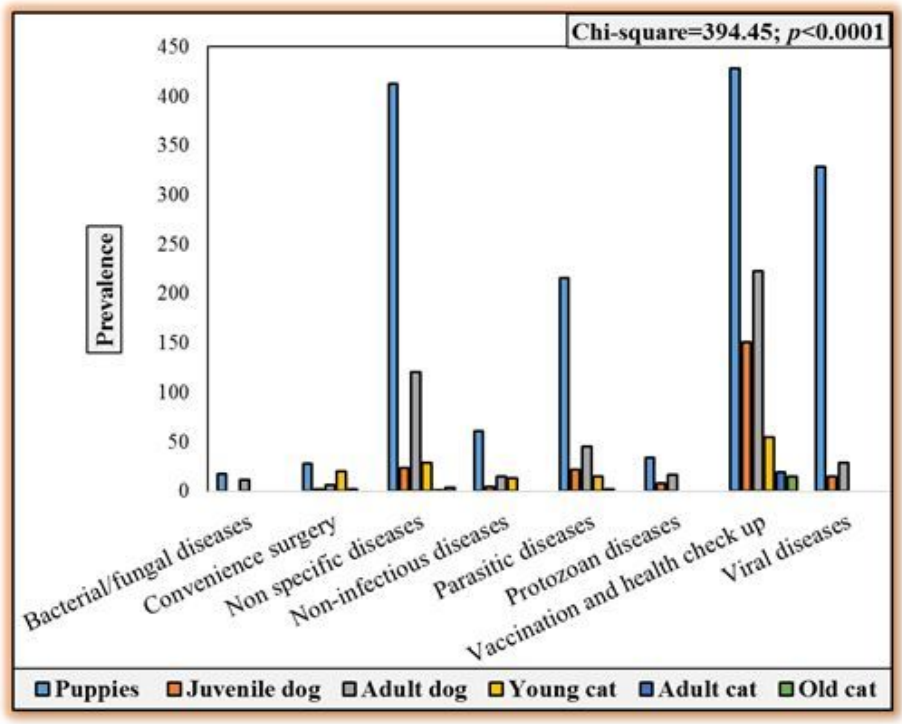

Figure 4

Breed distribution for 175 cats and 2222 dogs examined at private veterinary practices 